\title{
pH 7.35 to 7.45- Mathematical Reasoning of Treatment Principle Based on Yin Yang Wu Xing Theory in Traditional Chinese Medicine (II)
}

\author{
Yingshan Zhang \\ School of Statistics, Faculty of Economics and Management, East China Normal University, Shanghai, P. R. China
}

Email address:

yszhang@stat.ecnu.edu.cn

\section{To cite this article:}

Yingshan Zhang. pH 7.35 to 7.45- Mathematical Reasoning of Treatment Principle Based on Yin Yang Wu Xing Theory in Traditional Chinese Medicine (II). International Journal of Chinese Medicine. Vol. 4, No. 3, 2020, pp. 37-53. doi: 10.11648/j.ijcm.20200403.11

Received: January 13, 2017; Accepted: December 18, 2017; Published: July 13, 2020

\begin{abstract}
H}$ is useful in understanding disease. By using mathematical reasoning based on Yin Yang Wu Xing Theory in Traditional Chinese Medicine (TCM), this paper demonstrates that for the human blood pH value, the normal range of theory is [7.34539, 7.45461] nearly to [7.35, 7.45], and the center is 7.4. The first or second transfer law of human diseases changes according to the different human blood $\mathrm{pH}$ value whether in the normal range or not respectively. The treatment principle: "Don't have disease cure cure non-ill” (不治已病治未病) is abiding by the first or second transfer law of human diseases. Assume that the range of a human blood $\mathrm{pH}$ value is divided into four parts from small to large. Both second and third are for a healthy body. The treating works are the prevention or the treatment for a more serious relation disease which comes from the first transfer law; And both first and fourth are for an unhealthy body. The treating works are the prevention or the treatment for a more serious relation disease which comes from the second transfer law. Human disease treatment should protect and maintain the balance of two incompatibility relations: the loving relationship and the killing relationship. As an application, the generalized method of drug treatment of heart is given.
\end{abstract}

Keywords: Traditional Chinese Medicine (TCM), Yin Yang Wu Xing Theory, Steady Multilateral Systems, Incompatibility Relations, Side Effects Issue, Medical and Drug Resistance Problem

\section{Introduction}

The human blood $\mathrm{pH}$ value is useful in understanding the disease of human body's spleen organs. The human blood $\mathrm{pH}$ value is a general parameter linking together the complexity of relations between organ pairs, the organ itself, the capabilities for intervention reaction and self-protection of the body and mind as a whole, related to the environment, food, health and personal history, air, water, earth, climate, season, etc. $\mathrm{pH}$ is as useful in understanding disease as the average is in statistics, or as the expected value is in probability calculation.

The studies of Prof. Macro Pianesi, President of the International Association UN PUNTO MACROBIOTICO, began as a pioneer work continuing the work of Japanese Nyoiti Sakurazawa, known in the West as Georges Ohsawa, the founding father of Macrobiotics. For example, thanks to these studies and macrobiotic cooking, over 25, 000 disease patients have been cured in the last 8 years in the state of
Africa alone, even with understandable and interesting economic implications.

Macrobiotic diet therapy trials have started in other parts of the world as well, including China. Prof. Wang Bin (Director of the Department of Medical Matters, Tibetan and Ethnic Medicine Hospital, University of Traditional Chinese Medicine, Beijing), Dr. Weiguo Ma and Dr. Bing Xu (also from the University of Traditional Chinese Medicine, Beijing), and Dr. Yujing Quian (Doctor in Traditional Chinese Medicine at Capital Normal University), who reported their experiences in a conference held in Italy on October 18, 2010.

Mario Pianesi never ceases to thank the Ancient Chinese for the depth of their thinking, and for the value they give to food in their reflections and observations. Among others, Prof. Mario Pianesi identifies an important indicator for human health: the value of blood $\mathrm{pH}$, which, under normal conditions, ranges from 7.35 to 7.45 and the center is 7.4. Outside this range (acid:Yin condition; alkaline: Yang condition), disease appears. Almost always, when there is disease, the condition 
of the blood $\mathrm{pH}$ value is a Yin condition, little is a Yang condition. There are a lot of evidences (e.g., experimental identification for probability and real applications) to support this viewpoint, such as, Shirakabe et al. [1], Kaur et al. [2], Aly et al. [3], Intven et al. [4], Patel et al. [5], Handman et al. [6], Natalia et al. [7], Barfod et al. [8], Zhu et al. [9], Zhu et al. [10], Robert et al. [11], Mona et al. [12], Saritas et al. [13], Stevens et al. [14], and so on.

The body begins to activate the necessary mechanisms to restore this parameter to its appropriate range. If the body is unable to restore optimal $\mathrm{pH}$ levels, the disease may become chronic and lead to dire consequences.

Zhang et al. [15-23] have started a great interest and admired works for Traditional Chinese Medicine (TCM), where, through mathematical reasoning, they demonstrate the presence of incompatibility relations, which are predominant in daily life, yet absent in traditional Aristotelian Western logic.

Many people as Western person are beyond all doubt the Yin Yang Wu Xing theory is superior to the traditional true-false logic, which does not contemplate incompatibility relations, which Zhang [17-19] has expertly explained from a mathematical standpoint.

The work Zhang [15-19] has started, allows many people like Western person to think of a true re-foundation of mathematical language, to make it a better suited tool for the needs of mankind and the environment. Even so, Zhang [17, 18 ] also brings to light the difficulty of establishing the values of both the intervention reaction coefficients $\rho_{1}, \rho_{2}$ and the self-protection coefficient $\rho_{3}$ as parameters with due accuracy.

In this paper, the introduction of a parameter such as a blood $\mathrm{pH}$ value will be suggested, in order to facilitate the understanding and the calculation of these values. This paper ventures to suggest this with all due to respect, because it be believed that the path Zhang [15-19] has started, in such an understandable way from the mathematical point of view, will be very useful for all mankind searching for tools to understand the mechanisms of life.

The article proceeds as follows. Section 2 contains a parameter model and basic theorems, in order to explain the intervention reaction coefficients $\rho_{1}, \rho_{2}$ and the self-protection coefficient $\rho_{3}$ through the introduction of a parameter model to study the normal range of a human blood $\mathrm{pH}$ value, while the first or second transfer law of human diseases is demonstrated in Section 3, proved through the concept of both relation diseases and the relationship analysis of steady multilateral systems. Furthermore, if the range of a human blood $\mathrm{pH}$ value is divided into four parts from small to large, for the body in every part, the prevention or treatment method of human diseases as the treatment principle of TCM is given respectively in Section 4. As an application, the generalized method of drug treatment of heart is given in Section 5 and conclusions are drawn in Section 6.

\section{Parameter Model and Basic Theorems}

The concepts and notations in Zhang [17] are used start and still.

Let $\varphi=(\sqrt{5}-1) / 2=0.61803399$ be the gold number. Denoted $\rho_{0}=0.5897545123$, namely healthy number. It is because the healthy number $\rho_{0}$ can make the healthy balance conditions $\rho_{1}=\rho_{3}, \rho_{2}=\rho_{1} \rho_{3}$ and $1-\rho_{2} \rho_{3}=\rho_{1}+\rho_{2} \rho_{3}$ hold if $\rho_{1}=\rho_{0}, \rho_{2}=\rho_{0}^{2}$ and $\rho_{3}=\rho_{0}$.

Assume that $\rho_{0}^{\prime}=0.68232780$, namely unhealthy number. It is because under a poor self-protection ability, the unhealthy number $\rho_{0}^{\prime}$ can make the following unhealthy balance conditions hold:

$$
\begin{aligned}
& \rho_{1}-\rho_{3}=\rho_{3}=\rho_{0}^{\prime} / 2=0.34116390, \\
& \rho_{2}-\rho_{1} \rho_{3}=\rho_{1} \rho_{3}=\left(\rho_{0}^{\prime}\right)^{2} / 2=0.23278561 \\
& 1-\rho_{2} \rho_{3}=\rho_{1}+\rho_{2} \rho_{3}
\end{aligned}
$$

if $\rho_{1}=\rho_{0}^{\prime}, \rho_{2}=\left(\rho_{0}^{\prime}\right)^{2}=0.46557123$ and $\rho_{3}=\frac{1}{2} \rho_{0}^{\prime}$. Thus $\rho_{0}<\varphi<\rho_{0}^{\prime}$.

A parameter model of a human blood $\mathrm{pH}$ value in a mathematical sense based on Yin Yang Wu Xing Theory of TCM is reintroduced by using the functions $\lambda(x)$ and $\rho(x)$ of human blood $\mathrm{pH}$ value $x$ described as follows.

Let $x \in(7,7.8)$ be a human blood $\mathrm{pH}$ value, where the values 7 and 7.8 are the minimum and maximum acceptable the blood pH value. Denoted the value 7.4 is the target as the expectation of the human blood $\mathrm{pH}$ value. Define a function $\lambda(x)$ of the blood $\mathrm{pH}$ value $x$ in below:

$$
\begin{gathered}
\lambda(x)=\frac{|x-7.4|}{(7.8-x)(x-7)}, \forall x \in(7,7.8) \\
= \begin{cases}\frac{7.4-x}{(7.8-x)(x-7)}, & 7<x<7.4 ; \\
\frac{x-7.4}{(7.8-x)(x-7)}, & 7.4 \leq x<7.8 .\end{cases}
\end{gathered}
$$

A parameter model is considered as

$$
\rho(x)=\frac{1 / 2}{\lambda(x)+1 / 2}, \forall x \in(7,7.8)
$$

Theorem 2.1. Under model (2), the following statements hold.

(1) The one that $0<\rho(x)=\frac{1 / 2}{\lambda(x)+1 / 2} \leq 1$ is equivalent to the other that

$$
0 \leq \lambda(x)=\frac{1-\rho(x)}{2 \rho(x)}<+\infty
$$

where $\lambda(x)$ is a monotone decreasing function of $x$ if $x \in(7,7.4)$ or a monotone increasing function of $x$ if $x \in[7.4,7.8)$; and $\rho(x)$ is a monotone decreasing function 
of $\lambda(x)$ if $\lambda(x) \in[0,+\infty)$; and $\lambda(x)$ is a monotone decreasing function of $\rho(x)$ if $\rho(x) \in(0,1]$.

(2) If $1 \geq \rho(x) \geq \rho_{0}$, then

$\lambda(x)=\frac{1-\rho(x)}{2 \rho(x)} \leq \frac{1-\rho_{0}}{2 \rho_{0}}=\rho_{0}^{2} \leq \rho(x)^{2} \leq 1 ;$

$\frac{\lambda(x)}{\rho(x)}=\frac{1-\rho(x)}{2 \rho(x)^{2}} \leq \frac{1-\rho_{0}}{2 \rho_{0}^{2}}=\rho_{0} \leq \rho(x) \leq 1 ;$ and

$\frac{\lambda(x)}{\rho(x)^{2}}=\frac{1-\rho(x)}{2 \rho(x)^{3}} \leq \frac{1-\rho_{0}}{2 \rho_{0}^{3}}=1$.

(3) If $0<\rho(x)<\rho_{0}$, then

$\lambda(x)=\frac{1-\rho(x)}{2 \rho(x)}>\frac{1-\rho_{0}}{2 \rho_{0}}=\rho_{0}^{2}>\rho(x)^{2}>0 ;$

$\frac{\lambda(x)}{\rho(x)}=\frac{1-\rho(x)}{2 \rho(x)^{2}}>\frac{1-\rho_{0}}{2 \rho_{0}^{2}}=\rho_{0}>\rho(x)>0 ;$ and

$\frac{\lambda(x)}{\rho(x)^{2}}=\frac{1-\rho(x)}{2 \rho(x)^{3}}>\frac{1-\rho_{0}}{2 \rho_{0}^{3}}=1$.

(4) Taking $0<\rho_{1}=\rho(x)<\rho_{0}, \rho_{2}=\rho(x)^{2}$ and $\rho_{3}=c \rho(x)$ where $0 \leq c \leq 1$, there are

$\rho_{1}-\rho_{3}=\rho(x)(1-c) \geq 0, \rho_{2}-\rho_{1} \rho_{3}=\rho(x)^{2}(1-c) \geq 0$, and $\left(\rho_{1}+\rho_{2} \rho_{3}\right)=\rho(x)+c \rho(x)^{3}<1-\rho_{2} \rho_{3}=1-c \rho(x)^{3}$,

where $\left|\left(\rho_{1}+\rho_{2} \rho_{3}\right)-\left(1-\rho_{2} \rho_{3}\right)\right|>2(1-c) \rho_{0}^{3}=(1-c) 0.41024$.

(5) Taking $1 \geq \rho_{1}=\rho(x) \geq \rho_{0}, \rho_{2}=\rho(x)^{2}$ and $\rho_{3}=c \rho(x)$ where $0 \leq c \leq 1$, there are

firstly, $\rho_{1}-\rho_{3}=\rho(x)(1-c) \geq 0, \rho_{2}-\rho_{1} \rho_{3}=\rho(x)^{2}(1-c) \geq 0$ and $\left(\rho_{1}+\rho_{2} \rho_{3}\right)=\rho(x)+c \rho(x)^{3} \geq 1-\rho_{2} \rho_{3}=1-c \rho(x)^{3}$

$$
\text { if } 1 \geq c \geq \frac{1-\rho(x)}{2 \rho(x)^{3}}=\frac{\lambda(x)}{\rho(x)^{2}} \geq 0 \text {; }
$$

secondly,

$\rho_{1}-\rho_{3}=\rho(x)(1-c)>\rho(x) / 2, \rho_{2}-\rho_{1} \rho_{3}=\rho(x)^{2}(1-c)>\rho(x)^{2} / 2$ and $\left(\rho_{1}+\rho_{2} \rho_{3}\right)=\rho(x)+c \rho(x)^{3}<1-\rho_{2} \rho_{3}=1-c \rho(x)^{3}$ where $\left|\left(\rho_{1}+\rho_{2} \rho_{3}\right)-\left(1-\rho_{2} \rho_{3}\right)\right| \leq\left(\rho_{0}^{\prime}\right)^{3}=0.31767$ if $0 \leq c<\frac{1-\rho(x)}{2 \rho(x)^{3}}=\frac{\lambda(x)}{\rho(x)^{2}} \leq \frac{1}{2}$ in which $1>\rho(x) \geq \rho_{0}^{\prime}$;

thirdly,

$$
\begin{aligned}
& \rho_{1}-\rho_{3}=\rho(x)(1-c) \geq \rho(x) / 2, \\
& \rho_{2}-\rho_{1} \rho_{3}=\rho(x)^{2}(1-c) \geq \rho(x)^{2} / 2
\end{aligned}
$$

and $\left(\rho_{1}+\rho_{2} \rho_{3}\right)=\rho(x)+c \rho(x)^{3}<1-\rho_{2} \rho_{3}=1-c \rho(x)^{3}$ where $\left|\left(\rho_{1}+\rho_{2} \rho_{3}\right)-\left(1-\rho_{2} \rho_{3}\right)\right| \leq 2 \rho_{0}^{3}=0.41024$ if $0 \leq c \leq \frac{1}{2}<\frac{1-\rho(x)}{2 \rho(x)^{3}}=\frac{\lambda(x)}{\rho(x)^{2}} \leq 1$ in which $\rho_{0} \leq \rho(x)<\rho_{0}^{\prime}$; finally,

$$
\begin{aligned}
& \rho_{1}-\rho_{3}=\rho(x)(1-c)<\rho(x) / 2, \\
& \rho_{2}-\rho_{1} \rho_{3}=\rho(x)^{2}(1-c)<\rho(x)^{2} / 2
\end{aligned}
$$

and $\left(\rho_{1}+\rho_{2} \rho_{3}\right)=\rho(x)+c \rho(x)^{3}<1-\rho_{2} \rho_{3}=1-c \rho(x)^{3}$

where $\quad\left|\left(\rho_{1}+\rho_{2} \rho_{3}\right)-\left(1-\rho_{2} \rho_{3}\right)\right|<\left(\rho_{0}^{\prime}\right)^{3}=0.31767 \quad$ if $\frac{1}{2}<c<\frac{1-\rho(x)}{2 \rho(x)^{3}}=\frac{\lambda(x)}{\rho(x)^{2}} \leq 1$ in which $\rho_{0} \leq \rho(x)<\rho_{0}^{\prime}$.

In particular, when $c$ is nearly to $1 / 2$, there are

$$
\begin{aligned}
& \rho_{1}-\rho_{3}=\rho(x)(1-c) \rightarrow \rho(x) / 2, \\
& \rho_{2}-\rho_{1} \rho_{3}=\rho(x)^{2}(1-c) \rightarrow \rho(x)^{2} / 2
\end{aligned}
$$

and the following statements hold.

(a). The absolute value $\left|\left(\rho_{1}+\rho_{2} \rho_{3}\right)-\left(1-\rho_{2} \rho_{3}\right)\right|$ is nearly to 0 if $0<c<\frac{1-\rho(x)}{2 \rho(x)^{3}}=\frac{\lambda(x)}{\rho(x)^{2}} \leq \frac{1}{2}$ in which $1>\rho(x) \geq \rho_{0}^{\prime}$.

(b). The value $\left[\left(\rho_{1}+\rho_{2} \rho_{3}\right)-\left(1-\rho_{2} \rho_{3}\right)\right]$ is included in the interval $\left[-\rho_{0}^{3}=-0.20512,0\right)$ if $0<c \leq \frac{1}{2}<\frac{1-\rho(x)}{2 \rho(x)^{3}}=\frac{\lambda(x)}{\rho(x)^{2}} \leq 1 \quad$ in which $\rho_{0} \leq \rho(x)<\rho_{0}^{\prime}$.

(c). The value $\left[\left(\rho_{1}+\rho_{2} \rho_{3}\right)-\left(1-\rho_{2} \rho_{3}\right)\right]$ is included in the interval $\left[-\rho_{0}^{3}=-0.20512,0\right)$ if $\frac{1}{2}<c<\frac{1-\rho(x)}{2 \rho(x)^{3}}=\frac{\lambda(x)}{\rho(x)^{2}} \leq 1$ in which $\rho_{0} \leq \rho(x)<\rho_{0}^{\prime}$. \#

Corollary 2.1. Under model (2), the following statements hold.

(1) For any $0<d<1$, there is an unique solution $u \in(7,7.4)$ and there is also an unique solution $v \in(7.4,7.8)$, such that

$$
\begin{aligned}
& \lambda(7.4)=0 \leq \lambda(x)=\frac{1-\rho(x)}{2 \rho(x)} \leq \lambda(u)=\lambda(v)=(1-d) /(2 d), \\
& \rho(u)=\rho(v)=d \leq \rho(x)=\frac{1 / 2}{\lambda(x)+1 / 2} \leq 1=\rho(7.4) .
\end{aligned}
$$

(2) The condition $x \in[7.35,7.45]$ is equivalent to each of the following conditions:

$$
\begin{aligned}
& \lambda(7.4)=0 \leq \lambda(x)=\frac{1-\rho(x)}{2 \rho(x)} \leq \lambda(7.35)=\lambda(7.45)=0.31743, \\
& \rho(7.35)=\rho(7.45)=0.61167 \leq \rho(x)=\frac{1 / 2}{\lambda(x)+1 / 2} \leq 1=\rho(7.4) .
\end{aligned}
$$

(3) The condition $x \in[7.35129,7.44871]$ is equivalent to each of the following conditions:

$$
\begin{aligned}
& \lambda(7.4)=0 \leq \lambda(x)=\frac{1-\rho(x)}{2 \rho(x)} \leq \lambda(7.35129)=\lambda(7.44871)=0.30902, \\
& \rho(7.35129)=\rho(7.44871)=\varphi \leq \rho(x)=\frac{1 / 2}{\lambda(x)+1 / 2} \leq 1=\rho(7.4) .
\end{aligned}
$$

(4) The condition $x \in[7.34539,7.45461]$ is equivalent to each of the following conditions: 


$$
\begin{aligned}
& \lambda(7.4)=0 \leq \lambda(x) \leq \lambda(7.34539)=\lambda(7.45461)=\rho_{0}^{2}=0.34781, \\
& \rho(7.34539)=\rho(7.45461)=\rho_{0} \leq \rho(x)=\frac{1 / 2}{\lambda(x)+1 / 2} \leq 1=\rho(7.4) .
\end{aligned}
$$

(5) The condition $x \in[7.36307,7.43693]$ is equivalent to each of the following conditions:

$$
\begin{aligned}
& \lambda(7.4)=0 \leq \lambda(x) \leq \lambda(7.36307)=\lambda(7.43693)=\left(\rho_{0}^{\prime}\right)^{2} / 2=0.23279 \\
& \rho(7.36307)=\rho(7.43693)=\rho_{0}^{\prime} \leq \rho(x)=\frac{1 / 2}{\lambda(x)+1 / 2} \leq 1=\rho(7.4) . \#
\end{aligned}
$$

Remark 1. In west, through experiment or through practice observation, many researchers [1-14] have obtained the normal range of human blood $\mathrm{pH}$ value as $x \in[7.35,7.45]$. But in TCM, from Yin Yang Wu Xing Theory, Zhang [17] has already determined: $\rho_{0} \leq \rho_{1} \leq 1$ for the normal range of a healthy body. Assume that $\rho_{1}=\rho(x), \rho_{2}=\rho(x)^{2}$ and $\rho_{3}=c \rho(x)$ where $0 \leq c \leq 1$ for a human body which has the capabilities of both intervention reaction and self-protection, from Corollary 2.1, the condition $\rho_{0} \leq \rho_{1}=\rho(x) \leq 1$ is equivalent to $x \in[7.34539,7.45461]$. In other words, in Theory of TCM, the normal range of human blood $\mathrm{pH}$ value is considered as $x \in[7.34539,7.45461]$, nearly to $x \in[7.35,7.45]$, and the center is 7.4. Of course, little difference of the two intervals which makes the diagnosis of disease as a result, there may be no much difference. In fact, TCM uses the rule $\rho_{0} \leq \rho_{1} \leq 1$ from Yin Yang Wu Xing Theory instead of the normal range of a human blood $\mathrm{pH}$ value. The equivalence of Corollary 2.1 shows that TCM is The scientific.

Zhang [17] has already determined: a body is said a healthy mathematical complex system when the intervention reaction coefficient $\rho_{1}$ satisfies $1 \geq \rho_{1} \geq \rho_{0}$. In logic and practice, it's reasonable that $\rho_{1}+\rho_{2}$ is near to 1 if the input and output in a complex system is balanced, since a mathematical output subsystem at least is absolutely necessary other subsystems of all consumption. In case: $\rho_{1}+\rho_{2}=1$, all the energy for intervening mathematical complex subsystem can transmit to other mathematical complex subsystems which have neighboring relations or alternate relations with the intervening mathematical complex subsystem. The condition $1 \geq \rho_{1} \geq \rho_{0}$ can be satisfied that $\left(1-\rho_{2} \rho_{3}\right) \leq\left(\rho_{1}+\rho_{2} \rho_{3}\right), \quad \rho_{2}=\rho_{1} \rho_{3}$ and $\rho_{3}=\rho_{1}$ for a mathematical complex system since $\rho_{1}+\rho_{2}=1$ implies $\rho_{1}=\varphi \approx 0.61803 \geq \rho_{0}$. In this case, $\rho_{2}=\varphi^{2} \approx 0.38197 \geq \rho_{0}^{2}$. If this assumptions is set up, then the intervening principle: "Real disease with a healthy body is to rush down its son and virtual disease with a healthy body is to fill its mother" based on "Yin Yang Wu Xing" theory in image mathematics by Zhang and Shao [20], is quite reasonable.

But, in general, the ability of self-protection is often insufficient for an usual mathematical complex system, i.e., $\rho_{3}$ is small. A common standard is $\rho_{3}=\frac{1-\rho_{1}}{2 \rho_{2}} \approx \frac{1}{2}$ which comes from the balance condition $\left(1-\rho_{2} \rho_{3}\right)=\left(\rho_{1}+\rho_{2} \rho_{3}\right)$ of the loving relationship if $\rho_{1}+\rho_{2} \approx 1$. In other words, there is a principle which all losses are bear in mathematical complex system. Thus the general condition is often $\rho_{1} \approx 0.61803 \geq \rho_{3} \approx 0.5 \geq \rho_{2} \approx 0.38197$.

Interestingly, they are all near to the golden numbers. It is the idea to consider the unhealthy number $\rho_{0}^{\prime}=0.68232780$ since the poor condition of self-protection ability $\rho_{3}=\rho_{0}^{\prime} / 2=0.34116390$ can make the poor healthy balance conditions hold

$$
\begin{aligned}
& \rho_{1}-\rho_{3}=\rho_{3}=\rho_{0}^{\prime} / 2=0.34116390, \\
& \rho_{2}-\rho_{1} \rho_{3}=\rho_{1} \rho_{3}=\left(\rho_{0}^{\prime}\right)^{2} / 2=0.23278561 \\
& 1-\rho_{2} \rho_{3}=\rho_{1}+\rho_{2} \rho_{3}
\end{aligned}
$$

if $\rho_{1}=\rho_{0}^{\prime}, \quad \rho_{2}=\left(\rho_{0}^{\prime}\right)^{2}=0.46557123$ and $\rho_{3}=\rho_{0}^{\prime} / 2=0.34116390$.

By Theorem 2.1 and Corollary 2.1, $x \in[7.35,7.45]$ implies

$$
1 \geq \rho_{1}=\rho(x) \geq 0.61167=\rho(7.35)=\rho(7.45) .
$$

And $x \in[7.35129,7.44871]$ implies

$$
1 \geq \rho_{1}=\rho(x) \geq \varphi=\rho(7.35129)=\rho(7.44871) .
$$

And $x \in[7.34539,7.45461]$ implies

$$
1 \geq \rho_{1}=\rho(x) \geq \rho_{0}=\rho(7.34539)=\rho(7.45461),
$$

where $\lambda(7.34539)=\lambda(7.45461)=\frac{1-\rho_{0}}{2 \rho_{0}}=\rho_{0}^{2}$ since $\left(1-\rho_{0}^{3}\right)=\left(\rho_{0}+\rho_{0}^{3}\right)$.

And $x \in[7.36307,7.43693]$ implies

$$
1 \geq \rho_{1}=\rho(x) \geq \rho_{0}^{\prime}=\rho(7.36307)=\rho(7.43693),
$$

where $\lambda(7.36307)=\lambda(7.43693)=\frac{1-\rho_{0}^{\prime}}{2 \rho_{0}^{\prime}}=\frac{\left(\rho_{0}^{\prime}\right)^{2}}{2}$ since

$\left(1-\rho_{0}^{\prime}\right)=\left(\rho_{0}^{\prime}\right)^{3}$.

The last one is the healthy interval in a person's self-protection ability poor conditions. The interval range than the normal human body health requirements too strict, only the first three interval ranges are considered as a normal human body health. If keep two decimal places, then the first three intervals are the same as $x \in[7.35,7.45]$. This shows that range $x \in[7.35,7.45]$ is stable. The interval as the normal range of human blood $\mathrm{pH}$ value may be also appropriate. To conservative estimates, the interval with the length of the largest of the first three range intervals, i.e., $x \in[7.34539,7.45461]$, is used as the theoretical analysis of the normal range.

In fact, the range $x \in[7.34539,7.45461]$ is better than the range $x \in[7.35,7.45]$. It is because the range 
$x \in[7.34539,7.45461] \quad$ satisfies the healthy balance conditions $\rho_{1}=\rho_{3}, \rho_{2}=\rho_{1} \rho_{3}$ and $\left(1-\rho_{2} \rho_{3}\right) \leq\left(\rho_{1}+\rho_{2} \rho_{3}\right)$ if $\rho_{1}=\rho(x), \rho_{2}=\rho(x)^{2}$ and $\rho_{3}=c \rho(x) \rightarrow \rho_{1}$. In other words, the parameter $\rho_{1}=\rho(x) \geq \rho_{0}$ or the range $x \in[7.34539,7.45461]$ is the healthy running condition of both the killing relationship and the loving relation at the same time. But neither are the others. The human blood $\mathrm{pH}$ value must be precise calculation to keep at least 5 decimal places can ensure correct because of its sensitivity to the diagnosis of disease.\#

Remark 2. Western Medicine is different from TCM because the TCM has a concept of Chi or Qi (气) as a form of energy. As the energy concept, to say that one organ or subsystem of the human body is not running properly (or disease, abnormal), it is equivalent to say that the energy deviation from the average of the organ is too large, the high (real disease) or the low (virtual disease).

For the normal range of blood $\mathrm{pH}$ value of a human body as $x \in[7.34539,7.45461]$, in TCM, if $x>7.45461$, the body is considered as a real disease since the human blood $\mathrm{pH}$ value is too high; if $x<7.34539$, the body is considered as a virtual disease since the human blood $\mathrm{pH}$ value is too low.

Thus TCM identifies an important indicator for a human body's health: the human blood $\mathrm{pH}$ value, which, under normal conditions, ranges from 7.34539 to 7.45461 . Outside this range (acid: Yin condition; alkaline: Yang condition), disease appears. Almost always absolutely, when there is virtual disease, the condition of a human blood $\mathrm{pH}$ value is a Yin condition; when there is real disease, the condition of a human blood $\mathrm{pH}$ value is a Yang condition.

But there do not exist these concepts of both real diseases and virtual diseases in Western Medicine.\#

Remark 3. Obviously, when applying the hypothesis of Theorem 2.1 and Corollary 2.1 to other fields rather than human body's health, it is necessary to identify a global parameter in each field, such that it is able to yield a general Yin or Yang condition. And it is in relation to the average behavior of the studied phenomenon, and it maintains the equations at a sufficiently simple level of writing and application.

In fact, let $x \in(\min , \max )$ where the values $\min$ and max are the minimum and maximum acceptable the index $x$. Denoted the value $t_{0}$ is the target as the expectation of the index $x$ such that $\rho\left(t_{0}\right)=1$. In Eqs. (1) and (2), replace $7,7.8,7.4$ by $\min , \max , t_{0}$, respectively. The equivalent condition of a healthy body $\rho_{0} \leq \rho_{1}=\rho(x) \leq 1$ can be obtained as $x \in[u, v]$, $\min <u<t_{0}<v<\max$, where

$$
\begin{aligned}
& \rho(u)=\rho(v)=\rho_{0} \leq \rho_{1}= \\
& \rho(x)=(1 / 2) /[\lambda(x)+(1 / 2)] \leq 1=\rho\left(t_{0}\right)
\end{aligned}
$$

and

$$
\begin{aligned}
& \lambda\left(t_{0}\right)=0 \leq \lambda(x)=(1-\rho(x)) /(2 \rho(x)) \\
& \leq \rho_{0}^{2}=\lambda(u)=\lambda(v)=\rho(u)^{2}=\rho(v)^{2} \\
& \leq \rho_{2}=\rho(x)^{2} \leq \rho_{1}=\rho(x) \leq 1 . \#
\end{aligned}
$$

\section{Relationship of Steady Multilateral Systems}

\subsection{Energy Changes of a Steady Multilateral System}

In order to apply the reasoning to other fields rather than human body's health, Zhang [17] has started a steady multilateral system imitating a human body. A most basic steady multilateral system is as follows.

Theorem 3.1. (Zhang and Shao [20]) For each element $x$ in a steady multilateral system $V$ with two incompatibility relations, there exist five equivalence classes below:

$$
\begin{aligned}
& X=\{y \in V \mid y \sim x\}, X_{S}=\{y \in V \mid x \rightarrow y\}, X_{K}=\{y \in V \mid x \Rightarrow y\}, \\
& K_{X}=\{y \in V \mid y \Rightarrow x\}, S_{X}=\{y \in V \mid y \rightarrow x\},
\end{aligned}
$$

which the five equivalence classes have relations in Figure 1.\#

It can be proved by Theorem 3.2 that the steady multilateral system in Theorem 3.1 is the reasoning model of Yin Yang Wu Xing in TCM if there is an energy function $\varphi(*)$ satisfying

$$
\varphi\left(X_{K}\right) \geq \varphi\left(X_{S}\right) \geq \varphi(X) \geq \varphi\left(K_{X}\right) \geq \varphi\left(S_{X}\right)
$$

which is called Yin Yang Wu Xing model, denoted by $V^{5}$.

The Yin Yang Wu Xing model can be written as follows:

Define $V_{0}=X, V_{1}=X_{S}, V_{2}=X_{K}, V_{3}=K_{X}, V_{4}=S_{X}$, corresponding to wood, fire, earth, metal, water, respectively, and assume

$$
V=V_{0}+V_{1}+V_{2}+V_{3}+V_{4}
$$

where $V_{i} \cap V_{j}=\varnothing, \forall i \neq j$. And take $\Re=\left\{R_{0}, R_{1}, \cdots, R_{4}\right\}$ satisfying

$$
\begin{aligned}
& R_{r}=\sum_{i=0}^{4} V_{i} \times V_{\bmod (i+r, 5)}, r \in\{0,1, \cdots, 4\} \\
& R_{i} * R_{j} \subseteq R_{\bmod (i+j, 5)}
\end{aligned}
$$

where $V_{i} \times V_{j}=\left\{(x, y): x \in V_{i}, y \in V_{j}\right\}$ is the Descartes product in set theory and $R_{i} * R_{j}=\left\{(x, y): \exists u \in V\right.$ such that $\left.(x, u) \in R_{i},(u, y) \in R_{j}\right\}$ is the relation operation. The relation multiplication of $*$ is isomorphic to the addition of module 5. Then $V^{5}$ is a steady multilateral system with one equivalent relation $R_{0}$ and two incompatibility relations $R_{1}=R_{4}^{-1}$ and $R_{2}=R_{3}^{-1}$ where 
the relation inverse operation is

$$
R_{i}^{-1}=\left\{(x, y):(y, x) \in R_{i}\right\}, \forall i \in\{0,1, \cdots, 4\} .
$$

The Yin and Yang means the two incompatibility relations and the $\mathrm{Wu}$ Xing means the collection of five disjoint classification of $V=V_{0}+V_{1}+V_{2}+V_{3}+V_{4}$.

Figure 1 in Theorem 3.1 is the figure of Yin Yang Wu Xing theory in Ancient China. The steady multilateral system $V$ with two incompatibility relations is equivalent to the logic architecture of reasoning model of Yin Yang Wu Xing theory in Ancient China. What describes the general method of complex systems can be used in human complex systems.

By non-authigenic logic of TCM, i.e., a logic which is similar to a group has nothing to do with the research object by Zhang and Shao [20], in order to ensure the reproducibility such that the analysis conclusion can be applicable to any complex system, a logical analysis model which has nothing to do with the object of study should be chosen. The Tao model of Yin and Yang is a generalized one which means that two is basic. But the Tao model of Yin Yang is simple in which there is not incompatibility relation. The analysis conclusion of Tao model of Yin Yang cannot be applied to an incompatibility relation model. Thus the Yin Yang Wu Xing model with two incompatibility relations of Theorem 3.1 will be selected as the logic analysis model in this paper.

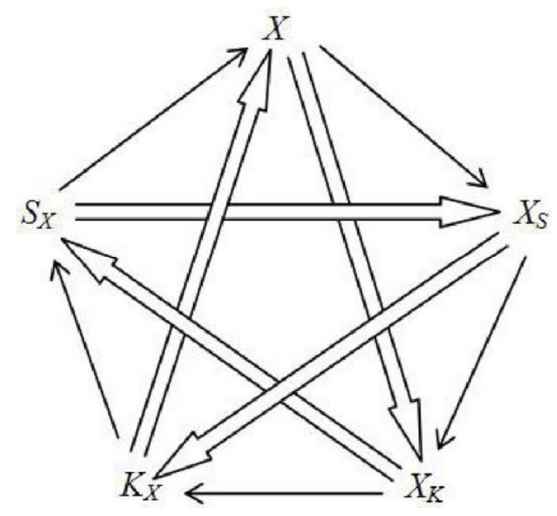

Figure 1. Finding Yin Yang Wu Xing Model.

Western Medicine is different from TCM because the TCM has a concept of Chi or $Q \mathrm{i}$ (气) as a form of energy of steady multilateral systems. It is believed that this energy exists in all things of steady multilateral systems (living and non-living) including air, water, food and sunlight. Chi is said to be the unseen vital force that nourishes steady multilateral systems' body and sustains steady multilateral systems' life. It is also believed that an individual is born with an original amount of $C h i$ at the beginning of steady multilateral systems' life and as a steady multilateral system grows and lives, the steady multilateral system acquires or attains $C h i$ or energy from "eating" and "drinking", from "breathing" the surrounding "air" and also from living in its environment. The steady multilateral system having an energy is called the anatomy system or the first physiological system.

And the first physiological system also affords Chi or energy for the steady multilateral system's meridian system (Jing-Luo (经络) or Zang Xiang (藏象)) which forms a parasitic system of the steady multilateral system, called the second physiological system of the steady multilateral system. The second physiological system of the steady multilateral system controls the first physiological system of the steady multilateral system. A steady multilateral system would become ill or dies if the Chi or energy in the steady multilateral system is imbalanced or exhausted, which means that $\rho_{1}=\rho(x) \rightarrow 0, \rho_{2}=\rho(x)^{2} \rightarrow 0$ and $\rho_{3}=c \rho(x) \rightarrow 0$.

For example, in TCM, a human body as the first physiological system of the steady multilateral system following the Yin Yang $\mathrm{Wu}$ Xing theory was classified into five equivalence classes in Zhang [17] as follows:

wood $(X)=\{$ liver, bravery, soul, ribs, sour, east, spring, birth\};

xiang-fire $\left(X_{S}^{x}\right)=$ ppericardium, the triple energizer, nerve, the blood, bitter taste, the south, summer, growth $\}$;

earth $\left(X_{K}\right)=\{$ spleen, stomach, willing, meat, sweetness, center, long summer, combined $\}$;

metal $\left(K_{X}\right)=\{$ lung, large intestine, boldness, fur, spicy, west, autumn, accept $\}$;

water $\left(S_{X}\right)=\{$ kidney, bladder, ambition, bone, salty, the north, winter, hiding $\}$.

jun-fire $\left(X_{S}^{j}\right)=\{$ heart, small intestine, nerve, the blood, bitter taste, all directions, whole-year, whole-growth $\}$;

fire $\left(X_{S}\right)=$ xiang-fire $\left(X_{S}^{x}\right) \cup$ jun-fire $\left(X_{S}^{j}\right)$.

There is only one of both loving and killing relations between every two classes. General neighboring is loving, alternate is killing.

In every category of internal, think that they are with an equivalent relationship, between each two of their elements there is a force of similar material accumulation of each other. It is because their pursuit of the goal is the same, i.e., follows the same "Axiom system". It can increase the energy of the class at low cost near to zero if they accumulate together. Any nature material activity follows the principle of maximizing so energy or minimizing the cost. In general, the size of the force of similar material accumulation of each other is smaller than the size of the loving force or the killing force in a stable complex system. The stability of any complex system first needs to maintain the equilibrium of the killing force and the loving force. The key is the killing force. For a stable complex system, if the killing force is large, i.e., $\rho_{3}=c \rho(x)$ becomes larger by Theorem 3.3 below, which needs positive exercise, then the loving force is also large such that the force of similar material accumulation of each other is also large. They can make the complex system more stable. If the killing force is small, i.e., $\rho_{3}=c \rho(x)$ becomes smaller by Theorem 3.3 below, which means little exercise, then the loving force is also small such that the force of similar material accumulation of each other is also small. They can make the complex system becoming unstable.

The second physiological system of the steady multilateral system controls the first physiological system of the steady multilateral system, abiding by the following rules. 
Attaining Rule: The second physiological system of the steady multilateral system will work by using Attaining Rule, if the first physiological system of the steady multilateral system runs normally. The work is in order to attain the Chi or energy from the first physiological system of the steady multilateral system by mainly utilizing the balance of the loving relationship of the first physiological system.

In mathematics, suppose that the body of $X$ is healthy. If $X$ is intervened, then the second physiological system will attain the $C h i$ or energy from $X$ directly.

Suppose that the body of $X$ is unhealthy. If $X$ is intervened, then the second physiological system will attain the $C h i$ or energy from $X$ indirectly. If virtual $X$ is intervened, it will attain the $C h i$ or energy (Yang energy) from the son $X_{S}$ of $X$. If real $X$ is intervened, it will attain the $C h i$ or energy (Yin energy) from the mother $S_{X}$ of $X$.\#

Affording Rule: The second physiological system of the steady multilateral system will work by using Affording Rule, if the first physiological system of the steady multilateral system runs hardly. The work is in order to afford the Chi or energy for the first physiological system of the steady multilateral system, by mainly protecting or maintaining the balance of the killing relationship of the steady multilateral system, to drive the first physiological systems will begin to run normally.

In mathematics, suppose that the body of $X$ is healthy. The second physiological system doesn't afford any Chi or energy for the first physiological system.

Suppose that the body of $X$ is unhealthy and the capability of self-protection is lack, i.e., $\rho_{3}=c \rho(x) \rightarrow 0$ and $0<\rho_{1}=\rho(x)<\rho_{0}$. The second physiological system will afford the $C h i$ or energy for $X$ directly, at the same time, affording the $C h i$ or energy for other subsystem, in order to protect or maintain the balance of the killing relationship, abiding by the intervening principle of "Strong inhibition of the same time, support the weak", such that the capability of self-protection is restored, i.e, $\rho_{3}=c \rho(x)>0$ and $\rho_{1}=\rho(x) \geq \rho_{0}$, to drive the first physiological system beginning to work.\#

The Chi or energy is also called the food hereafter for simply. In order to get the food, by Attaining Rule, the second physiological system must make the first physiological system intervened, namely exercise. It is because only by intervention in the first physiological system, the second physiological system can be to get food.

Energy concept is an important concept in Physics. Zhang [17] introduces this concept to the steady multilateral systems. And image mathematics by Zhang and Shao [20] uses these concepts to deal with the steady multilateral system diseases (mathematical index too high or too low). In mathematics, a steady multilateral system is said to have an Energy (or Dynamic) if there is a non-negative function $\varphi(*)$ which makes every subsystem meaningful of the steady multilateral system. Similarly to Zhang [17], unless stated otherwise, any equivalence relation is the liking relation, any neighboring relation is the loving relation, and any alternate relation is the killing relationship.

Suppose that $V$ is a steady multilateral system having energy, then $V$ in the steady multilateral system during a normal operation, its energy function for any subsystem of the steady multilateral system has an average (or expected value in Statistics), this state is called as normal when the energy function is nearly to the average. Normal state is the better state.

That a subsystem of the steady multilateral system is not running properly (or disease, abnormal) is that the energy deviation from the average of the subsystems is too large, the high (real disease) or the low (virtual disease).

In addition to study these real or virtual diseases, TCM is also often considered a kind of relation diseases. The relation disease is defined as the relation of two sick subsystems. In general, a relation disease is less serious if the relation satisfies one of both the loving relationship and the killing relationship of the steady multilateral system. In this case, in general, the human blood $\mathrm{pH}$ value $x \in[7.34539,7.45461]$ which means $\rho_{0} \leq \rho_{1}=\rho(x) \leq 1$. This relation disease is less serious because this relation disease can not undermine the loving order or the killing order of the steady multilateral system. The less serious relation disease can make the intervention increasing the sizes of both the intervention reaction coefficients $\rho_{1}, \rho_{2}$ and the self-protection coefficient $\rho_{3}$.

But the relation disease is more serious if the relation not only doesn't satisfy the killing relationship of the steady multilateral system, but also can destroy the killing order, i.e., there is an incest order. In this case, in general, the human blood $\mathrm{pH}$ value $x \notin[7.34539,7.45461]$ which means $0<\rho_{1}=\rho(x)<\rho_{0}$. This relation disease is more serious because the relation disease can destroy the killing order of the steady multilateral system if the disease continues to develop. The more serious can make the sizes of both the intervention reaction coefficients $\rho_{1}, \rho_{2}$ and the self-protection coefficient $\rho_{3}$ decreasing response to intervention.

There are also many relation diseases which cannot destroy the loving order or the killing order of the steady multilateral system although the relation doesn't satisfy strictly the loving relationship or the killing relationship of the steady multilateral system, i.e., there is not an incest order. These relation diseases are called rare since they are hardly occurrence for a healthy body.

The purpose of intervention is to make the steady multilateral system return to the normal state. The method of intervention is to increase or decrease the energy of a subsystem.

What kind of intervening should follow the principle to treat it? Western medicine emphasizes directly treatments on disease subsystem after the disease of subsystem has occurred, but the indirect intervening of oriental medicine is required before the disease of subsystem will occur. In mathematics, which is more reasonable?

Based on this idea, many issues are worth further discussion. For example, if an intervening has been implemented to a disease subsystem before the disease of subsystem will occur, what relation disease will be less serious which does not need to be intervened? what relation disease will be more serious which needs to be intervened? 


\subsection{Kinds of Relation Diseases of Steady Multilateral Systems}

For a steady multilateral system $V$ imitating a human body with two incompatibility relations, suppose that the subsystems $X, X_{S}, X_{K}, K_{X}, S_{X}$ are the same as those defined in Theorem 3.1. Then the relation diseases can be decomposed into the following classes:

Definition 3.1. (involving (相及) and infringing (相犯)) Suppose that both $X$ and $X_{S}$ having the loving relationship fall ill. Consider a relation disease occurred between $X$ and $X_{S}$.

The relation disease between $X$ and $X_{S}$ is called less serious if $X$ is a virtual disease and so is $X_{S}$ at the same time. The less serious relation disease between virtual $X$ and virtual $X_{S}$ is also called the mother's disease involving in her son. The mother is the cause of the disease.

The relation disease between $X$ and $X_{S}$ is also called less serious if $X$ is real disease and so is $X_{S}$ at the same time. The less serious relation disease between real $X$ and real $X_{S}$ is also called the son's disease infringing upon its mother. The son is the cause of disease.

The relation disease between $X$ and $X_{S}$ is called rare if $X$ is a real disease but $X_{S}$ is a virtual disease at the same time, or if $X$ is a virtual disease but $X_{S}$ is a real disease at the same time. The rare relation disease implies that they cannot destroy the loving order although real $X$ cannot love virtual $X_{S}$ or virtual $X$ cannot love real $X_{S}$.\#

Definition 3.2. (multiplying (相乘) and insulting (相侮)) Suppose that both $X$ and $X_{K}$ having the killing relationship fall ill. Consider a relation disease occurred between $X$ and $X_{K}$.

The relation disease between $X$ and $X_{K}$ is called less serious if $X$ is a real disease and $X_{K}$ is a virtual disease at the same time. The less serious relation disease between $X$ and $X_{K}$ is also called a multiplying relation.

The relation disease between $X$ and $X_{K}$ is called more serious if $X$ is a virtual disease but $X_{K}$ is a real disease at the same time. The more relation disease between $X$ and $X_{K}$ is also called an insulting relation. It means that $X$ has been harmed by $X_{K}$ through the method of incest.

The relation disease between $X$ and $X_{K}$ is called rare if $X$ is a real disease and so is $X_{K}$ at the same time, or if $X$ is a virtual disease and so is $X_{K}$ at the same time. The rare relation disease implies that they cannot destroy the killing order from $X$ to $X_{K}$ although real $X$ cannot kill real $X_{K}$ or virtual $X$ cannot kill virtual $X_{K}$.

The relation disease between $X, X_{K}$ and $K_{X}$ is called more serious if $X$ is a real disease, and $X_{K}$ is a virtual disease but $K_{X}$ is also a virtual disease at same time, i.e., not only real $X$ multiplies in virtual $X_{K}$, but also insults virtual $K_{X}$ by using the method of incest. It is because the energy of real $X$ is too high. The more serious relation disease between $X, X_{K}$, and $K_{X}$ is also called a multiplying-insulting (乘侮) relation.

The relation disease between $X, X_{K}$, and $S_{X}$ is called more serious if $X$ is a real disease, and $X_{K}$ is a virtual disease but $S_{X}$ is also a real disease at same time, i.e., not only real $X$ multiplies in virtual $X_{K}$, but also real $S_{X}$ insults virtual $X_{K}$ by using the method of incest. It is because the energy of virtual $X_{K}$ is too low. The more serious relation disease between $X, X_{K}$, and $S_{X}$ is also called a multiplying-insulting (乘侮) relation.

Only the more serious relation disease can destroy the killing relationship order of Yin Yang Wu Xing system. All the therapeutic principles need to prevent the more serious relation disease occurrence in the first place. \#

The disease of multiplying-insulting relation will result in more than three subsystems falling-ill. Generally, three or more subsystems falling-ill, it will be hard to cure. Therefore, the multiplying-insulting relation disease should be avoided as much as possible. In Chinese words, it is that "Again and again, not only to the repeated to four” (只有再一再二, 没有再三再四)-Allow one or two subsystems fall ill, but Don't allow three or four subsystems fall ill.

\subsection{Transfer Law of Diseases of Steady Multilateral Systems with a Healthy Body}

Suppose that a steady multilateral system $V$ imitating a human body having energy function $\varphi\left({ }^{*}\right)$ is normal or healthy. Let $x$ be the human blood $\mathrm{pH}$ value of $V$. Assume that $\rho_{1}=\rho(x), \rho_{2}=\rho(x)^{2}$, and $\rho_{3}=c \rho(x)$ where $0 \leq c \leq 1$ and $\rho(x)$ is defined in Eqs. (1) and (2). The healthy body means that the conditions $\rho_{0} \leq \rho(x) \leq 1$ and $0<c \leq 1$ hold. By Corollary 2.1, it is equivalent to the normal range $x \in[7.34539,7.45461]$ or the healthy condition $\rho_{1}+\rho_{2} \rho_{3} \geq 1-\rho_{2} \rho_{3}$. That $c \rightarrow 0$ implies that the body is without the ability of self-protection, i.e., $\rho_{3}=c \rho(x) \rightarrow 0$. Of course, the body cannot be healthy. It is because

$$
\begin{aligned}
& \rho_{1}+\rho_{2} \rho_{3}=\rho(x)+c \rho(x)^{3} \rightarrow \rho(x)<1 \\
& \leftarrow 1-c \rho(x)^{3}=1-\rho_{2} \rho_{3}, \forall x \neq 7.4
\end{aligned}
$$

such that the healthy condition $\rho_{1}+\rho_{2} \rho_{3} \geq 1-\rho_{2} \rho_{3}$ cannot hold.

By using Corollary 2.1 and Theorems 2.1 and 3.1, the following Theorem 3.2 can be obtained as the transfer law of occurrence and change of diseases with a healthy body.

Theorem 3.2. Suppose that a steady multilateral system is healthy. Let the human blood pH value be $x \in[7.34539,7.45461]$ which is equivalent to the conditions: $\rho_{0} \leq \rho_{1}=\rho(x) \leq 1=\rho(7.4)$ and $0<c \leq 1$.

In this case, almost always, the less serious relation disease will occur and change. If the disease continues to develop, the 
change can make a more serious relation disease occur.

The occurrence and change of diseases with a healthy body has its transfer law: The first occurrence and change of the loving relationship and the killing relationship after the loving relationship disease. In other words, the following statements are true.

(1) If a subsystem $X$ of a steady multilateral system $V$ falls a virtual disease, the transfer law is the first occurrence of the virtual disease of the mother $S_{X}$ of $X$ with a less serious relation disease between virtual $S_{X}$ and virtual $X$, and secondly the real disease of the bane $K_{X}$ of $X$ after the virtual disease of $S_{X}$ with a less serious relation disease between real $K_{X}$ and virtual $X$, and thirdly the real disease of the prisoner $X_{K}$ of $X$ with a more serious relation disease between virtual $X$ and real $X_{K}$, and fourthly the virtual disease of the son $X_{S}$ of $X$ with a less serious relation disease between virtual $X$ and virtual $X_{S}$, and finally the new remission virtual disease of the subsystem $X$ itself, and for the next round of disease transmission, until disease rehabilitation.

(2) If a subsystem $X$ of a steady multilateral system $V$ encounters a real disease, the transfer law is the first occurrence of the real disease of the son $X_{S}$ of $X$ with a less serious relation disease between real $X$ and real $X_{S}$, and secondly the virtual disease of the prisoner $X_{K}$ of $X$ after the real disease of $X_{S}$ with a less serious relation disease between real $X$ and virtual $X_{K}$, and thirdly the virtual disease of the bane $K_{X}$ of $X$ with a more serious relation disease between virtual $K_{X}$ and real $X$, and fourthly the real disease of the mother $S_{X}$ of $X$ with a less serious relation disease between real $S_{X}$ and real $X$, and finally the new abated real disease of the subsystem $X$ itself, and for the next round of disease transmission, until disease rehabilitation.

All first transfer laws of diseases with a healthy body are summed up as Figures 2 and 3.\#

Remark 4. Theorem 3.2 is called the transfer law of occurrence and change of diseases with a healthy body, simply, the first transfer law.

For a real disease, the first transfer law is along the loving relationship order transmission as follows:

$$
\begin{aligned}
& \text { real } X \stackrel{\text { less }}{\rightarrow} \text { real } X_{S} \stackrel{\text { rare }}{\rightarrow} \text { virtual } X_{K} \\
& \text { more } \\
& \rightarrow \text { virtual } K_{X} \stackrel{\text { rare }}{\rightarrow} \text { real } S_{X} \stackrel{\text { less }}{\rightarrow} \text { real } X .
\end{aligned}
$$

For a virtual disease, the first transfer law is against the loving relationship order transmission as follows:

$$
\begin{aligned}
& \text { virtual } X \stackrel{\text { less }}{\leftarrow} \text { virtual } S_{X} \stackrel{\text { rare }}{\leftarrow} \text { real } K_{X} \\
& \text { more } \quad \text { real } X_{K} \stackrel{\text { rare }}{\leftarrow} \text { virtual } X_{S} \stackrel{\text { less }}{\leftarrow \text { virtual } X .}
\end{aligned}
$$

The transfer relation of the first transfer law running is the loving relationship, denoted by $\rightarrow$.

The running condition of the first transfer law is both $\left(\rho_{1}+\rho_{2} \rho_{3}\right) \geq\left(1-\rho_{2} \rho_{3}\right)$ and $\rho_{3}=c \rho(x)>0$. By Theorem 2.1 and Corollary 2.1 , the running condition is nearly equivalent to both $\rho_{0} \leq \rho_{1}=\rho(x) \leq 1$ and $0<c \leq 1$. The best-state condition of the first transfer law is $\rho_{3}=c \rho(x) \rightarrow \rho(x)=\rho_{1}$ where $c \rightarrow 1$ which is the best state of $\rho_{3}$ for the healthy body. To follow or utilize the running of the first transfer law is equivalent to the following method. For dong so, it is to protect and maintain the loving relationship. The method can strengthen both that the value $\left(\rho_{1}+\rho_{2} \rho_{3}\right)=\left(\rho(x)+c \rho(x)^{3}\right)$ tends to be large and that the value $\left(1-\rho_{2} \rho_{3}\right)=\left(1-c \rho(x)^{3}\right)$ tends to be small, at the same time. In other words, the treatment can make all of both $\rho(x)$ and $c$ tend to be large. It is because the running condition of the loving relationship $\left(\rho_{1}+\rho_{2} \rho_{3}\right) \geq\left(1-\rho_{2} \rho_{3}\right)$ is the stronger the use, which dues to $\rho_{1}=\rho(x)$ is the greater the use.

In other words again, if the treatment principle of the loving relationship diseases is to use continuously abiding by the first transfer law, then all of both the intervention reaction coefficients $\rho_{1}=\rho(x), \rho_{2}=\rho(x)^{2}$ and the coefficient of self-protection $\rho_{3}=c \rho(x)>0$ where $0<c \leq 1$ will tend to be the best state, i.e., $\rho(x) \rightarrow 1$ and $0<c \rightarrow 1$.\#

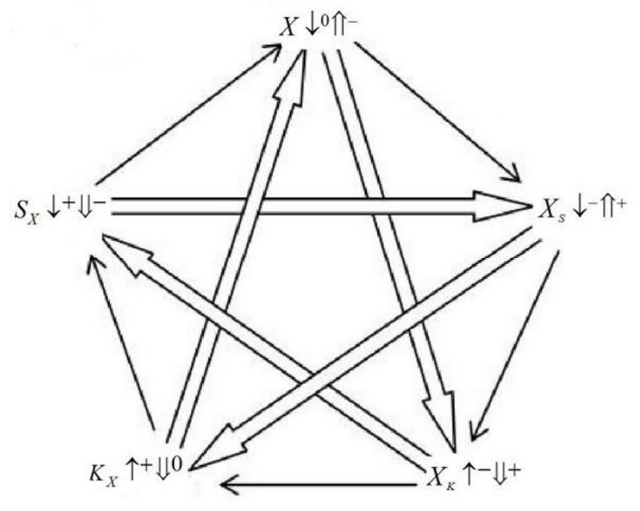

Figure 2. Transfer law of virtual-diseases for a healthy body.

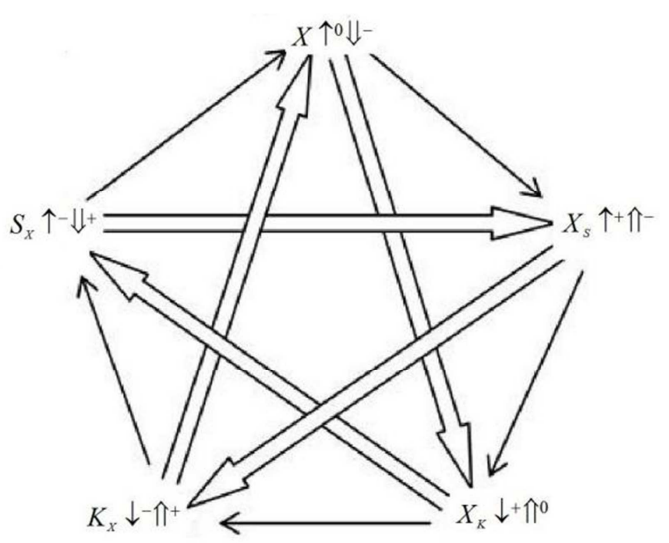

Figure 3. Transfer law of real-diseases for a healthy body.

Side effects of medical problems were the question: in the 
medical process, destroyed the balance of the normal systems which are not sick or intervened subsystems. The energy change of the intervened system is not the true side effects issue. The energy change is called the pseudo or non-true side effects issue since it is just the food of the second physiological system of the steady multilateral system for a healthy body by Attaining Rule. The best state of the self-protection coefficient, i.e., $\rho_{3}=c \rho(x) \rightarrow \rho(x)=\rho_{1}$, where $c \rightarrow 1$, implies the non-existence of any side effects issue if the treatment principle of TCM is used. Therefore any disease that causes side effects issue occurrence in the first place dues to the non-best state of self-protection ability, i.e., $\rho_{3}=c \rho(x)<\rho(x)=\rho_{1}$. To follow or utilize the running of the first transfer law can make both $\rho(x) \rightarrow 1$ and $0<c \rightarrow 1$ At this point, the paper advocates to follow or utilize the first transfer law. It is in order to avoid the side effects issue occurrence for a healthy body. \#

\subsection{Transfer Law of Diseases of Steady Multilateral Systems with an Unhealthy Body}

Suppose that a steady multilateral system $V$ imitating a human body having energy function $\varphi(*)$ is abnormal or unhealthy. Let $x$ be the human blood $\mathrm{pH}$ value of $V$. Assume that $\rho_{1}=\rho(x), \rho_{2}=\rho(x)^{2}$ and $\rho_{3}=c \rho(x) \quad$ where $0 \leq c \leq 1$, and $\rho(x)$ is defined in Eqs.(1) and (2). The unhealthy body means that the conditions $\rho_{0}>\rho_{1}=\rho(x)>0$ and $0 \leq c \leq 1$ hold, which is equivalent to the abnormal range $x \notin[7.34539,7.45461]$.

From Zhang and Shao [20], by using Corollary 2.1 and Theorems 2.1 and 3.1, the following Theorem 3.3 can be obtained as the transfer law of occurrence and change of diseases with an unhealthy body.

Theorem 3.3. Suppose that a steady multilateral system is unhealthy. Let the human blood $p H$ value be $x \notin[7.34539,7.45461]$ which is equivalent to the conditions:

$\rho_{0}>\rho_{1}=\rho(x)>0$ and $0 \leq c \leq 1$.

In this case, almost always, the rare relation disease will occur and change. If the disease continues to develop, the change can make a more serious relation disease occur.

The transfer of disease with an unhealthy body has its transfer law: Only there is the transfer of the killing relationship. In other words, the following statements are true.

(1) If a subsystem $X$ of a steady multilateral system $V$ falls a virtual disease, then the disease comes from the son $X_{S}$ of $X$. The transfer law is the first occurrence of the virtual disease of the prisoner $X_{K}$ of $X$ with a rare relation disease between virtual $X$ and virtual $X_{K}$, and secondly the virtual disease of the mother $S_{X}$ of $X$ after the virtual disease of $X_{K}$ with a rare relation disease between virtual $X_{K}$ and virtual $S_{X}$, and thirdly the virtual disease of the son $X_{S}$ of $X$ with a rare relation disease between virtual $S_{X}$ and virtual $X_{S}$, and fourthly the real disease of the bane $K_{X}$ of $X$ with a more serious relation disease between virtual $X_{S}$ and real $K_{X}$, and finally the new remission virtual disease of the subsystem $X$ itself with a less serious relation disease between real $K_{X}$ and virtual $X$, and for the next round of disease transmission, until disease rehabilitation.

(2) If a subsystem $X$ of a steady multilateral system $V$ encounters a real disease, then the disease comes from the mother $S_{X}$ of $X$. The transfer law is the first occurrence of the real disease of the bane $K_{X}$ of $X$ with a rare relation disease between real $K_{X}$ and real $X$, and secondly the real disease of the son $X_{S}$ of $X$ after the real disease of the bane $K_{X}$ of $X$ with a rare relation disease between real $X_{S}$ and real $K_{X}$, and thirdly the real disease of the mother $S_{X}$ of $X$ with a rare relation disease between real $S_{X}$ and real $X_{S}$, and fourthly the virtual disease of the prisoner $X_{K}$ of $X$ with a more serious relation disease between virtual $X_{K}$ and real $S_{X}$, and finally the new abated real disease of the subsystem $X$ itself with a less serious relation disease between real $X$ and virtual $X_{K}$, and for the next round of disease transmission, until disease rehabilitation.

All second transfer laws of diseases with an unhealthy body are summed up as Figures 4 and 5.\#

Remark 5. Theorem 3.3 is called the transfer law of occurrence and change of diseases with an unhealthy body, simply, the second transfer law.

For a virtual disease, the second transfer law is along the killing relationship order transmission as follows:

$$
\begin{aligned}
& \text { virtual } X \stackrel{\text { rare }}{\Rightarrow} \text { virtual } X_{K} \stackrel{\text { rare }}{\Rightarrow} \text { virtual } S_{X} \\
& \stackrel{\text { mare }}{\Rightarrow} \text { virtual } X_{S} \stackrel{\text { less }}{\Rightarrow} \text { real } K_{X} \stackrel{\text { virtual } X .}{\Rightarrow}
\end{aligned}
$$

For a real disease, the second transfer law is against the killing relationship order transmission as follows:

$$
\begin{aligned}
& \text { real } X \stackrel{\text { rare }}{\models} \text { real } K_{X} \stackrel{\text { rare }}{\Leftarrow} \text { real } X_{S} \\
& \stackrel{\text { rare }}{\models} \text { real } S_{X} \stackrel{\text { more }}{\models} \text { virtual } X_{K} \stackrel{\text { less }}{=} \operatorname{real} X .
\end{aligned}
$$

The transfer relationship of the second transfer law running is the killing relationship, denoted by $\Rightarrow$.

The running condition of the second transfer law is both $\left(\rho_{1}+\rho_{2} \rho_{3}\right)<\left(1-\rho_{2} \rho_{3}\right)$ and $\rho_{3}=c \rho(x) \geq 0$. By Theorem 2.1 and Corollary 2.1, the running condition is equivalent to both $\rho_{0}>\rho_{1}=\rho(x)>0$ and $\quad 1 \geq c \geq 0$. That $\rho_{3}=c \rho(x) \rightarrow 0$ means the lack of capability of self-protection. Of course, it is the basis condition of running the second transfer law.

The stopping condition of the second transfer law is both $\left(\rho_{1}+\rho_{2} \rho_{3}\right) \geq\left(1-\rho_{2} \rho_{3}\right)$ and $\rho_{3}=c \rho(x)>0$, which is the running condition of the first transfer law, or, the existence condition of capabilities of both intervention reaction and self-protection. To follow or utilize the running of the second transfer law is equivalent to the following method. For dong so, it is to protect and maintain the killing relationship of the 
steady multilateral system. The method can strengthen all of both $\rho_{1}-\rho_{3}=\rho(x)(1-c)$ and $\rho_{2}-\rho_{1} \rho_{3}=\rho(x)^{2}(1-c)$ tending to be small at the same time. In other words, the treatment can make $c$ tends to be large for fixed $\rho(x)>0$. It is because the transferring condition of the killing relation disease $\left(\rho_{1}+\rho_{2} \rho_{3}\right)<\left(1-\rho_{2} \rho_{3}\right)$ is the weaker the use, which dues to $\rho_{3}$ is the greater the use.

The way can make both $\rho_{1}-\rho_{3} \rightarrow 0$ and $\rho_{2}-\rho_{1} \rho_{3} \rightarrow 0$ at the same time such that the killing relation disease cannot be transferred. In other words again, if the treatment principle of the killing relationship diseases is to use continuously, abiding by the second transfer law, then the coefficient of self-protection will tend to be the occurrence state, i.e., $\rho_{3}=c \rho(x)>0 \quad$ where $\quad 1 \geq c \geq \frac{1-\rho(x)}{2 \rho(x)^{3}} \geq 0 \quad, \quad$ and the coefficients of intervention reaction also will tend to the healthy state, i.e., $\rho_{0} \leq \rho_{1}=\rho(x) \leq 1$, such that $\left(\rho_{1}+\rho_{2} \rho_{3}\right) \geq\left(1-\rho_{2} \rho_{3}\right)$.\#

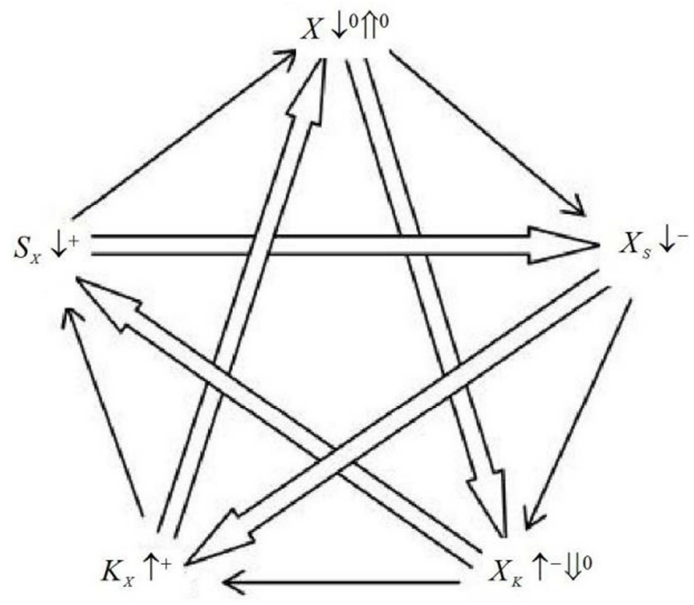

Figure 4. Transfer law of virtual diseases for an unhealthy body.

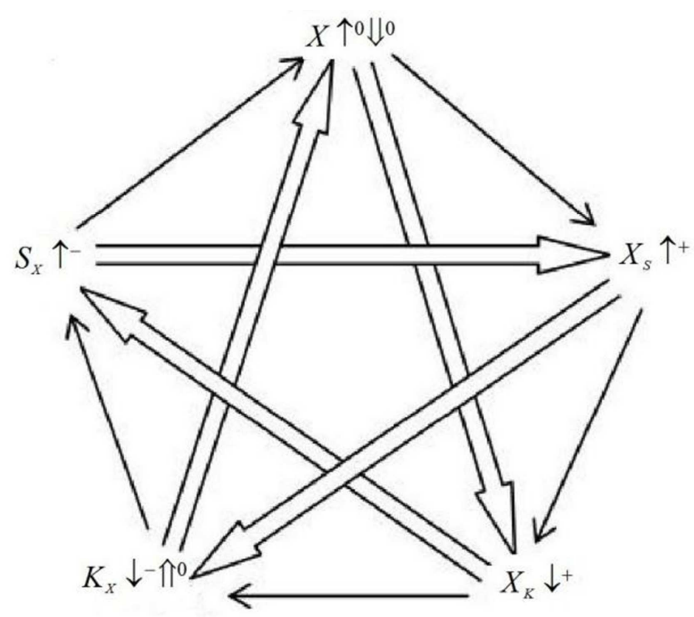

Figure 5. Transfer law of real diseases for an unhealthy body.

Medical and drug resistance problem is that such a question, beginning more appropriate medical treatment, but is no longer valid after a period. In the state

$$
\begin{aligned}
& \rho_{1}-\rho_{3}=\rho(x)(1-c) \rightarrow 0, \\
& \rho_{2}-\rho_{1} \rho_{3}=\rho(x)^{2}(1-c) \rightarrow 0
\end{aligned}
$$

by Theorem 3.2, any medical and drug resistance problem is non-existence if the treatment principle of TCM is used. But in the state

$$
\begin{aligned}
& \rho_{1}-\rho_{3}=\rho(x)(1-c) \rightarrow \rho(x), \\
& \rho_{2}-\rho_{1} \rho_{3}=\rho(x)^{2}(1-c) \rightarrow \rho(x)^{2},
\end{aligned}
$$

by Theorem 3.3, the medical and drug resistance problem is always existence, even if the treatment principle of TCM has been used. It is because virtual $X_{K}$ cannot kill real $S_{X}$ if $X$ is intervened by increasing its energy. In other words, the lack of capability of self-protection, i.e., $\rho_{3}=c \rho(x) \rightarrow 0$, implies the possible existence of a medical and drug resistance problem, although the treatment principle of TCM has been used. At this point, the paper advocates to follow or utilize the second transfer law. It is in order to prevent and avoid the medical and drug resistance problem occurrence for an unhealthy body.\#

\section{Treatment Principle of TCM}

In order to explain treatment principle of TCM, the changes in the blood $\mathrm{pH}$ value range are put to be divided into four parts from small to large. From Zhang and Shao [20], by Corollary 2.1 and Theorems 2.1, 3.2 and 3.3, it can be easily proved that the following theorem is true.

Theorem 4.1. Suppose that the subsystem $X$ of a steady multilateral system falls ill. Let $x$ be the human body blood $\mathrm{pH}$ value of the steady multilateral system. Denoted the parameters of the normal range as follows

$$
a=7.34539, b=7.45461, t_{0}=7.4 .
$$

Then the following statements are true.

(1) Suppose that $x<a$ as virtual, in which $X$ falls a virtual disease with an unhealthy body. The primary treatment is to increase the energy of the subsystem $X$ directly. And the secondary treatment is to increase the energy of the son $X_{S}$ of $X$, and at the same time, to decrease the energy of the prisoner $K_{X}$ of $X_{S}$.

(2) Suppose that $x \in\left[a, t_{0}\right)$ as virtual-normal, in which $X$ will fall an expected virtual disease with a healthy body. The primary treatment is to increase the energy of the mother subsystem $S_{X}$ of $X$ which is an indirect treating for $X$. And the secondary treatment is to increase the energy of $X$ itself, and at the same time, to decrease the energy of the prisoner $X_{K}$ of $X$.

(3) Suppose that $x \in\left[t_{0}, b\right]$ as real-normal, in which $X$ will encounter an expected real disease with a healthy body. The primary treatment is to decrease the energy of the son subsystem $X_{S}$ of $X$ which is an indirect treating for $X$. And the secondary treatment is to decrease the energy of $X$ itself, and at the same time, to increase the energy of the bane $K_{X}$ of 
$X$.

(4) Suppose that $x>b$ as real, in which $X$ encounters $a$ real disease with an unhealthy body. The primary treatment is to decrease the energy of the subsystem $X$ directly. And the secondary treatment is to decrease the energy of the mother $S_{X}$ of $X$, and at the same time, to increase the energy of the bane $X_{K}$ of $S_{X}$. \#

Remark 6. Treatment principle of Theorem 4.1 based on ranges of the human body blood $\mathrm{pH}$ value is called the treatment principle of TCM, since it is in order to protect and maintain the balance of two incompatibility relations: the loving relationship and the killing relationship.

For the unhealthy body where $x<a$ or $x>b$, the treatment principle is the method for doing so in the following:

The primary treatment is to increase or decrease the energy of $X$ directly corresponding to $x<a$ or $x>b$ respectively, and the secondary treatment is to increase the energy of $X_{S}$ or $X_{K}$ while to decrease the energy of $K_{X}$ or $S_{X}$, respectively.

The primary treatment is in order to protect and maintain the loving relationship, abiding by TCM's ideas "Virtual disease with an unhealthy body is to fill itself" and "Real disease with an unhealthy body is to rush down itself'. It is because the method for dong so is not only greatly medical diseases of their own, but also provides the pseudo side effects as the food for the second physiological system. The method is to promote the first physiological system running since the second physiological system controls the first physiological system. And it is also to improve the loving relationship to develop since the loving relationship mainly comes from the first physiological system. The loving relationship to develop can strengthen both that $\rho_{1}+\rho_{2} \rho_{3}=\rho(x)+c \rho(x)^{3}$ tends to be large and that $1-\rho_{2} \rho_{3}=1-c \rho(x)^{3}$ tends to be small at the same time. In other words, the way can make all of both $\rho(x)$ and $c$ tend to be large, at least, $c$ greater than zero for an unhealthy body and $\rho_{0} \leq \rho(x) \leq 1$, such that the body from unhealthy to healthy, or the first physiological system works, or, the occurrence of capability of self-protection, or, the running of the first transfer law, or, the stopping of the second transfer law.

The secondary treatment is in order to protect or maintain the killing relationship, abiding by TCM's ideas "Don't have disease cure cure non-ill" and "Strong inhibition of the same time, support the weak". By the second transfer law in Theorem 3.3, the more serious relation disease is the relation disease between virtual $X_{S}$ and real $K_{X}$, or between virtual $X_{K}$ and real $S_{X}$, respectively.

Abiding by TCM's idea "Don't have disease cure cure non-ill", it must be done to prevent or avoid the more serious relation disease between virtual $X_{S}$ and real $K_{X}$, or between virtual $X_{K}$ and real $S_{X}$ occurrence respectively.

Abiding by TCM's idea 'Strong inhibition of the same time, support the weak", it must be done to increase the energy of $X_{S}$ or $X_{K}$ while decrease the energy of $K_{X}$ or $S_{X}$ respectively.

The method for doing so can improve the killing relationship to develop since real $X_{S}$ or real $X_{K}$ can kill virtual $K_{X}$ or virtual $S_{X}$ respectively. The killing relationship to develop means that both $\rho_{1}-\rho_{3}=\rho(x)(1-c)$ and $\rho_{2}-\rho_{1} \rho_{3}=\rho(x)^{2}(1-c)$ tend to be small at the same time. In other words, the way can make, for fixed $\rho(x), c$ tending to be large, at least, greater than zero for an unhealthy body, such that the body from unhealthy to healthy, or the first physiological system works, or, the occurrence of capability of self-protection, or, the running of the first transfer law, or, the stopping of the second transfer law.

For the healthy body, where $x \in\left[a, t_{0}\right)$ or $x \in\left[t_{0}, b\right]$, the treatment principle is the method for doing so in the following:

The primary treatment is to increase or decrease the energy of $S_{X}$ or $X_{S}$ corresponding to $x \in\left[a, t_{0}\right)$ or $x \in\left[t_{0}, b\right]$ respectively, and the secondary treatment to increase the energy of $K_{X}$ or $X$ while to decrease the energy of $X$ or $X_{K}$, respectively.

The primary treatment is in order to protect and maintain the loving relationship, abiding by TCM's ideas "Virtual disease with a healthy body is to fill mother" and "Real disease with a healthy body is to rush down its son". It is because the method for dong so is not only greatly medical diseases of their own, but also provides the pseudo side effects as the food for the second physiological system. The method is to promote the first physiological system running since the second physiological system controls the first physiological system. And it is also to improve the loving relationship developing since the loving relationship mainly comes from the first physiological system. The loving relationship developing can strengthen both that $\rho_{1}+\rho_{2} \rho_{3}=\rho(x)+c \rho(x)^{3}$ tends to be large and that $1-\rho_{2} \rho_{3}=1-c \rho(x)^{3}$ tends to be small at the same time. In other words, using the way can make all of both $\rho(x)$ and $0<c$ tending to be large, the best, all equal to 1 for a healthy body, such that the capability of self-protection is in the best state, or, the non-existence of side effects issue, or, the non-existence of medical and drug resistance problem.

The secondary treatment is in order to protect or maintain the killing relationship, abiding by TCM's ideas "Don't have disease cure cure non-ill" and "Strong inhibition of the same time, support the weak". By the first transfer law, the more serious relation disease is the relation disease between virtual $K_{X}$ and real $X$ or between virtual $X$ and real $X_{K}$ corresponding to real $X$ or virtual $X$, respectively.

Abiding by TCM's idea "Don't have disease cure cure non-ill", it must be done to prevent and avoid the more serious relation disease between virtual $K_{X}$ and real $X$ or between virtual $X$ and real $X_{K}$ occurrence corresponding to real $X$ or virtual $X$ respectively.

Abiding by TCM's idea "Strong inhibition of the same time, support the weak", it must be done to increase the energy of $K_{X}$ or $X$ while decrease the energy of $X$ or $X_{K}$ respectively.

The method for doing so can improve the killing relationship developing since real $K_{X}$ or real $X$ can kill virtual 
$X$ or virtual $X_{K}$ respectively. The killing relationship developing also means that both $\rho_{1}-\rho_{3}=\rho(x)(1-c)$ and $\rho_{2}-\rho_{1} \rho_{3}=\rho(x)^{2}(1-c)$ tend to be small at the same time. In other words, using the way can make, for fixed $\rho(x), 0<c$ tending to be large, the best, equal to 1 for a healthy body, such that the capability of self-protection is in the best state, or, the non-existence of side effects, or, the non-existence of medical and drug resistance issue. \#

\section{Generalized Method of Drug Treatment of Heart}

Shirakabe et al. [1] have analyzed six hundred twenty-one consecutive patients with an arterial blood gas analysis (ABG) in acute heart failure (AHF) who were admitted to the intensive care unit of Chiba Hokusoh Hospital, Nippon Medical School between January 2000 and April 2011. The AHF belongs to "heart" in TCM, namely fire $\left(X_{S}\right)$ by Theorem 3.1.

The patients were assigned to an alkalosis group ( $n=99$, $p H>7.45)$, a normal acid-base balance group $(n=178,7.35 \leq$ $p H \leq 7.45)$, and an acidosis group $(n=344, p H<7.35)$ based on an ABG analysis in an emergency setting within 30 min of their admission. Basic survival data are stated in Table 1. Shirakabe et al. [1] reveal that there were 113 deaths among the 621 patients $(18.2 \%)$ within 2 years. The rate of death should be high since in TCM the "heart" subsystem is human nerve system controlling blood, whose high or low change of energy can rapidly lead to death. In other words, if consider the survival rate, it is the higher the better. Survival rates were considered for each of the three groups. A surprising fact was discovered. The alkalosis group is high, the acidosis group is middle and the normal acid-base balance group is low. It means that the directly treatment in west is right for the alkalosis or acidosis group and is not right for the normal acid-base balance group.

\subsection{A Real-normal Disease of Heart}

Watching Table 1 , for Normal $(n=178)$, the blood $\mathrm{pH}$ value $x$ included in the normal range [7.34539,7.45461] inside the normal range $[7.35,7.45]$ from rounding method. By (2) and (3) of Theorem 4.1, it means that the body is with a healthy body. But the fall-ill subsystem fire $\left(X_{S}\right)$ could get an expected real disease, also may make an expected virtual disease. By (2) and (3) of Theorem 4.1 again, the treatment for an expected real disease or virtual illness is completely different. Most afraid of those in TCM is the expected real disease as an expected deficiency disease cure or put the expected virtual disease as an expected real disease. In this case, in TCM, the general direct intervention methods cannot be used, only first by indirect way of treatment. Indirect treatment way as the primary treatment is to increase the energy of the mother subsystem wood $(X)$ of fire $\left(X_{S}\right)$ for an expected virtual disease commonly, or reduce the energy of the son subsystem earth $\left(X_{K}\right)$ of fire $\left(X_{S}\right)$ for an expected real disease. The auxiliary treatment should use as little as possible since mainly $\rho_{1}+\rho_{2} \rho_{3} \geq 1-\rho_{2} \rho_{3}$ which makes the auxiliary therapeutic effect is not obvious.

First consider an expected real disease situation where $7.4 \leq p H=x \leq 7.45461$. In TCM, it is called a real-normal disease of heart or a heat closed disease. With clinical gradual-change symptoms of the disease are as follows:

(1). High fever be agitated: Nerve faint, delirious speech, thirsty, sputum delay indicates, crimson greasy tongue, red or red-purple moss, pulse stimulation;

(2). Heart kidney Yin deficiency: Heat from the endogenous and interference in the heart, the heart tired, can not sleep; Virtual thermal disturbance nerves, much dream; Heat burning Yin makes Yin fluid not deck, dry mouth, pharynx dry; Pulse is strong.

By (3) of Theorem 4.1, the primary treatment is to decrease the energy of the son subsystem earth $\left(X_{K}\right)$ of fire $\left(X_{S}\right)$. And the secondary treatment is to decrease the energy of fire $\left(X_{S}\right)$ itself, and at the same time, to increase the energy of the bane water $\left(S_{X}\right)$ of fire $\left(X_{S}\right)$.

In fact, TCM also did just that. At this point, first of all, eat bitter-tasting medicines in order to reduce the spleen system of energy (to decrease the energy of the earth $\left(X_{K}\right)$ ). At the same time by means of adjuvant therapy slightly, that is to say: eat sweet medicine leakage of heart (to decrease the energy of fire $\left(X_{S}\right)$ itself), and bitterness medicine to fill the kidney energy (to increase the energy of the bane system water $\left(S_{X}\right)$ ).

\subsection{A Real Disease of Heart}

Watching Table 1, for Alkalosis ( $n=99)$, the blood $\mathrm{pH}$ value $x$ is greater than 7.45461 outside the normal range [7.35, 7.45] from rounding method. By (4) of Theorem 4.1, it means that the subsystem fire $\left(X_{S}\right)$ of the body with an unhealthy falls a real disease. In TCM, it is called a real disease of heart or an evil hot crack of pericardial disease which dues to the heat closed disease. With clinical gradual-change symptoms of the disease are as follows.

Because the real disease is according to the order of light to heavy, the real disease is the disease type which is heavier. It has three types, so start from (3), (4) to (5). The cases (1) and (2) are for the disease of the real-normal situation.

(3). Evil hot cracking heart: On hot stamping and head, dizziness; Mind may not be a Yin blood, forgetfulness; Red face lips, wrist arterial pulse is smooth;

(4). Kidney virtual: Kidney Yin deficiency can't go to glory in the ear, the tinnitus; Phlegm heat within the knot; Wrist arterial pulse is dynamic;

(5). Kidney be destroyed: Kidney can't nourish the mansion, the waist is sore, red tongue, less moss, wrist arterial pulse is count.

By (4) of Theorem 4.1, the primary treatment is to decrease the energy of the subsystem fire $\left(X_{S}\right)$ directly. And the secondary treatment is to decrease the energy of the mother wood $(X)$ of fire $\left(X_{S}\right)$, where $S_{\left(X_{S}\right)}=X$ in Figure 1, and at the same time, to increase the energy of the bane metal $\left(K_{X}\right)$ of $\operatorname{wood}(X)$. 
In fact, TCM did just that. For the drugs, these patients not only should eat the sweet medicines to rush down the energy of heart subsystem (to decrease the energy of the subsystem fire $\left(X_{S}\right)$ directly), but also should eat the sour medicines to rush down the energy of liver subsystem (to decrease the energy of the subsystem wood $(X))$ while eat the bitter-sour medicines to raise the energy of lung subsystem by nursing way (to increase the energy of the subsystem metal $\left(K_{X}\right)$ gradually).

The patients of alkalosis group might have experienced AHF for a few days and were associated with high In-hospital mortality (the highest $14(14.1 \%$ ) in Table 1). It is because for the patients of alkalosis group, in general or by Theorems 3.3 and 4.1, the energy of the liver subsystem wood $(X)$ is higher than normal for an unhealthy body with a real disease. The liver subsystem wood $(X)$ is human soul system controlling ribs, whose high energy can lead to death quickly. But for a long time, their survival rate is higher than Normal or Acidosis if they recovered discharge after directly treating. In Table 1, the survival rate of alkalosis group is the highest $42(42.4 \%)$ at 730 days of follow-up periods among the three groups, although the In-hospital mortality is the highest $14(14.1 \%)$. It means that the mainly directly intervene method in west is the right way for an unhealthy body with a real disease.

\subsection{A Virtual-Normal Disease of Heart}

Second consider an expected virtual disease situation where $7.34539 \leq p H=x<7.4$. In TCM, it is called a virtual-normal disease of heart or a fright of diseases or a disease of heart palpitations. With clinical gradual-change symptoms of the disease are as follows:

(a). Guilty timid: Guilty heart palpitation dues to panic, throbs, shortness of breath, spontaneous sweating, nervous fatigue, lack of power, insomnia, much dream, pale tongue, moss thin white, pulse fine less stimulation;

(b). The energy of heart and spleen two weak: Heart palpitations uneasy, insomnia, forgetfulness, face's complexion light white less blood's color, dizziness, fatigue, shortness of breath, easy to sweat, eat less, bosom frowsty, moss thin white, pulse is weak.

By (2) of Theorem 4.1, the primary treatment is to increase the energy of the mother subsystem wood $(X)$ of fire $\left(X_{S}\right)$. And the secondary treatment is to increase the energy of fire $\left(X_{S}\right)$ itself, and at the same time, to decrease the energy of the prisoner metal $\left(K_{X}\right)$ of fire $\left(X_{S}\right)$.

In fact, TCM also did just that. It is to eat spicy medicines in order to increase the energy of the liver subsystem (to increase the energy of the wood $(X)$ ). The auxiliary treatment is to eat the salty medicines to fill the energy of heart subsystem (to increase the energy of fire $\left(X_{S}\right)$ ) while eat again the spicy medicines in order to decrease the energy of the lung subsystem (to decrease the energy of the metal $\left(K_{X}\right)$ ).

The prognosis was different between the patients with normal acid-balance and alkalosis or acidosis in-hospital mortality (Normal 8(4.5\%), Alkalosis 14(14.1\%) and Acidosis
$32(9.3 \%)$ in Table 1). It is because for the patients with normal acid-balance, in general, by Theorems 3.2 and 4.1, the energy of the spleen subsystem earth $\left(X_{K}\right)$ is higher for a healthy body with an expected real disease or the energy of the liver subsystem wood $(X)$ is lower for a healthy body with an expected virtual disease. The liver subsystem wood $(X)$ is human soul system controlling ribs, whose low energy cannot lead to death quickly. And the spleen subsystem earth $\left(X_{K}\right)$ is the human willing system controlling meat, whose high energy also cannot lead to death quickly. But for a long time, their survival rate of normal acid-balance group is the lowest if they recovered discharge after directly treating. In Table 1, the survival rate of normal acid-balance group is the lowest $57(32.0 \%)$ at 730 days of follow-up periods among the three groups, although both ICU hospitalization and In-hospital mortality are all the lowest $7.5 \pm 12.9$ (days) and $8(4.5 \%$ ), respectively. It means that the mainly directly intervene method in west is not the right way for a healthy body.

\subsection{A Virtual Disease of Heart}

Watching Table 1 again, for Acidosis $(n=344)$, the blood $\mathrm{pH}$ value $x$ is smaller than 7.34539 outside the normal range $[7.35,7.45]$ from rounding method. By (1) of Theorem 4.1, it means that the subsystem fire $\left(X_{S}\right)$ of the body with an unhealthy falls a virtual disease. In TCM, it is called a virtual disease of heart or a possession of nerve-freezing disease which dues to the disease of heart palpitations. With clinical gradual-change symptoms of the disease are as follows.

Because the virtual disease is according to the order of light to heavy, the virtual disease is the disease type which is heavier. It has three types, so start from (c), (d) to (e). The cases (a) and (b) are for the disease of the virtual-normal situation.

(c). Blood stasis resistance: Palpitations froze and para, bosom is frowsty and heartache of attack, dark-purple face lips, tongue dark-purple or ecchymosis, wrist arterial pulse is not smooth and fine-astringent;

(d). Water vapour bullying heart: Nerves froze and para, bosom is frowsty and asthma, cough vomit a lot of bubble, phlegm delay, floating face, feet swollen, cannot lay down, dizzy, less urine, tongue's moss white greasy or white slippery, wrist arterial pulse is less dynamic and weak-sliding;

(e). Weak heart Yang deficiency: Heart throb froze and para, chest tightness and shortness of breath, cold and cold limbs, dizziness, pale face without blood's color, light tongue fat and whitish, wrist arterial pulse is heavy-fine-late and pause frequently or accidentally.

By (1) of Theorem 4.1, the primary treatment is to increase the energy of the subsystem fire $\left(X_{S}\right)$ directly. And the secondary treatment is to increase the energy of the son earth $\left(X_{K}\right)$ of fire $\left(X_{S}\right)$, where $\left(X_{S}\right)_{S}=X_{K}$ in Figure 1, and at the same time, to decrease the energy of the prisoner water $\left(S_{X}\right)$ of earth $\left(X_{K}\right)$, where $\left(X_{K}\right)_{K}=S_{X}$ in Figure 1 .

In fact, TCM also did just that. These patients not only should eat the salty medicines to fill the energy of heart subsystem (to increase the energy of the subsystem fire $\left(X_{S}\right)$ 
directly), but also should eat the sweet-salty medicines to fill or raise the energy of spleen subsystem by nursing way (to increase the energy of the subsystem earth $\left(X_{K}\right)$ ) while eat again the salty medicines to rush down the energy of kidney subsystem (to decrease the energy of the subsystem water $\left.\left(S_{X}\right)\right)$.

The prognosis was not different between the patients with acidosis and normal acid-balance in-hospital mortality (Acidosis 32(9.3\%) and Normal 8(4.5\%) in Table 1). It is because for the patients with acidosis AHF, in general or by Theorems 3.3 and 4.1, the energy of the spleen subsystem earth $\left(X_{K}\right)$ is lower than normal for an unhealthy body with a virtual disease. The spleen subsystem earth $\left(X_{K}\right)$ is human meaning system controlling meat, whose low energy cannot lead to death quickly. But for a long time, their survival rate is still a little bit higher than normal acid-balance rather than alkalosis AHF group if they recovered discharge after directly treating. In Table 1, the survival rate of acidosis AHF group is the middle state $117(35.0 \%)$ at 730 days of follow-up periods among the three groups, although both ICU hospitalization and Total hospitalization are all the highest $8.4 \pm 15.7$ (days) and $42.0 \pm 49.6$ (days), respectively. It means that the mainly directly intervene method in west is also the right way for an unhealthy body with a virtual disease, at least better than those for a healthy body.

Table 1. Comparison of arterial blood gas between three groups.

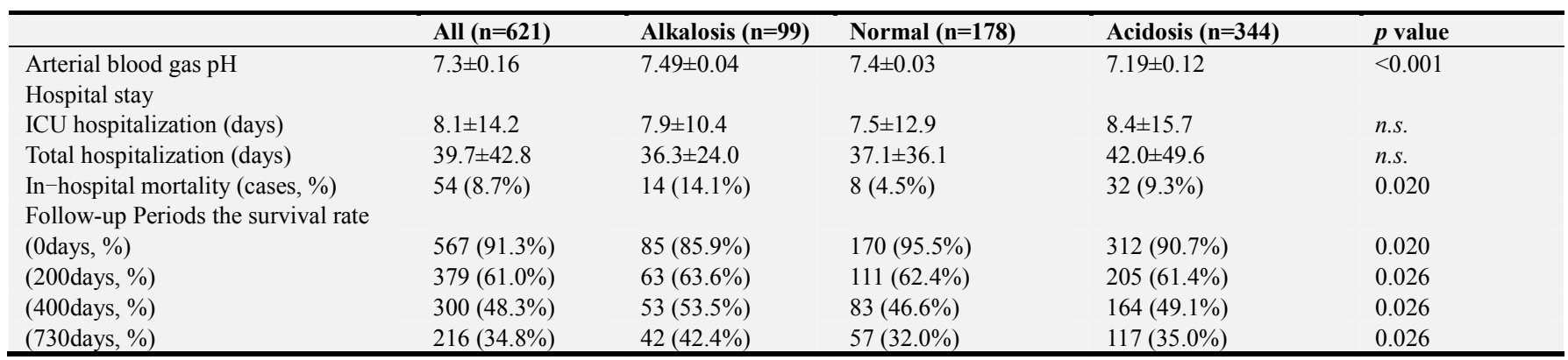

$p$ value: comparison with alkalosis, normal acid balance and acidosis groups by the Jonckheere-Terpstra test. n.s.: not specified; IUC: intensive care unit.

\section{Conclusions}

This work shows how to treat the diseases of a human body by using the human blood $\mathrm{pH}$ value $x$. For the human blood $\mathrm{pH}$ value, the normal range of theory is [7.34539, 7.45461] nearly to $[7.35,7.45]$, and the center is 7.4 . The first or second transfer law of human diseases changes according to the different human body's blood $\mathrm{pH}$ value whether in the normal range or not. For the normal range, the first transfer law in Theorem 3.2 runs; For the abnormal range, the second transfer law in Theorem 3.3 runs.

Assume that the range of human body's blood $p H$ value $x$ is divided into four parts from small to large. Both second and third are for a healthy body with an expected virtual or real disease respectively. The works are the prevention or the treatment for a more serious relation disease between virtual $X$ and real $X_{K}$ or between virtual $K_{X}$ and real $X$, respectively. Each of the two more serious relation diseases comes from the first transfer law in Theorem 3.2.

And both first and fourth are for an unhealthy body with a virtual or real disease respectively. The works are the prevention or the treatment for a more serious relation disease between virtual $X_{S}$ and real $K_{X}$ or between virtual $X_{K}$ and real $S_{X}$, respectively. Each of the two more serious relation diseases comes from the second transfer law in Theorem 3.3.

Human disease treatment should protect and maintain the balance or order of two incompatibility relations: the loving relationship and the killing relationship. The method for doing so can make the $\rho_{3}=c \rho(x)$ tending to be large. In other words, the treatment can make all of both $\rho(x)$ and $c$ tend to be large, at least, greater than zero for an unhealthy body; or, the best, tend to 1 for a healthy body.

The following way can make the capabilities of both intervention reaction and self-protection become in the best state, the non-existence of side effects issue, the non-existence of medical and drug resistance problem, and so on.

(1) Suppose that $x<a=7.34539$ as virtual, in which $X$ falls a virtual disease with an unhealthy body. In order to protect or maintain the loving relationship, abiding by TCM's idea "Virtual disease with an unhealthy body is to fill itself" (虚则补之), increase the energy of $X$ directly.

In order to protect or maintain the killing relationship, abiding by TCM's idea "Don't have disease cure cure non-ill" (不治已病治未病), do a preventive treatment for the more serious relation disease between virtual $X_{S}$ and real $K_{X}$. Through the intervening principle of "Strong inhibition of the same time, support the weak" (抑强扶弱), increase the energy of the son $X_{S}$ of $X$ while decrease the energy of the prisoner $K_{X}$ of $X_{S}$.

(2) Suppose that $x \in\left[a=7.34539, t_{0}=7.4\right) \quad$ as virtual-normal, in which $X$ falls an expected virtual disease with a healthy body. In order to protect or maintain the loving relationship, abiding by TCM's idea "Virtual disease with a healthy body is to fill its mother" (虚则补其母), increase the energy of the mother $S_{X}$ of $X$. The treating way is an indirect treating for $X$.

In order to protect or maintain the killing relationship, abiding by TCM's idea "Don't have disease cure cure non-ill" (不治已病治未病), do a preventive treatment for the more 
serious relation disease between virtual $X$ and real $X_{K}$. Through the intervening principle of "Strong inhibition of the same time, support the weak” (抑强扶弱), increase the energy of $X$ itself while decrease the energy of the prisoner $X_{K}$ of $X$.

(3) Suppose that $x \in\left[t_{0}=7.4, b=7.45461\right]$ as real-normal, in which $X$ falls an expected real disease with a healthy body. In order to protect or maintain the loving relationship, abiding by TCM's idea "Real disease with a healthy body is to rush down its son" (实则泄其子), decrease the energy of the son $X_{S}$ of $X$. The treating way is an indirect treating for $X$.

In order to protect or maintain the killing relationship, abiding by TCM's idea "Don't have disease cure cure non-ill" (不治已病治未病), do a preventive treatment for the more serious relation disease between virtual $K_{X}$ and real $X$. Through the intervening principle of "Strong inhibition of the same time, support the weak" (抑强扶弱), decrease the energy of $X$ itself while increase the energy of the bane $K_{X}$ of $X$.

(4)Suppose that $x>b=7.45461$ as real, in which $X$ falls a real disease with an unhealthy body. In order to protect or maintain the loving relationship, abiding by TCM's idea "Real disease with an unhealthy body is to rush down itself" (实则 泄之), decrease the energy of $X$ directly.

In order to protect or maintain the killing relationship, abiding by TCM's idea "Don't have disease cure cure non-ill” (不治已病治未病), do a preventive treatment for the more serious relation disease between virtual $X_{K}$ and real $S_{X}$. Through the intervening principle of "Strong inhibition of the same time, support the weak" (抑强扶弱), decrease the energy of the mother $S_{X}$ of $X$ while increase the energy of the bane $X_{K}$ of $S_{X}$.

\section{Acknowledgements}

Sincerely thanks Prof. Francesco Marchetti (Teacher at the Torelli High School, Fano, Italy; Member of the International Association UN PUNTO MACROBIOTICO, Founded by Prof. Mario Pianesi, Email: francescomarchetti56@gmail.com ) for his valuable advice.

This article has been repeatedly invited as reports, such as People's University of China in medical meetings, Shanxi University, Liaocheng University, Xuchang College, and so on. The work was supported by Specialized Research Fund for the Doctoral Program of Higher Education of Ministry of Education of China (Grant No. 200802691021).

\section{References}

[1] A. Shirakabe, N. Hata, N. Kobayashi, T. Shinada, K. Tomita, M. Tsurumi, M. Matsushita, H. Okazaki, Y. Yamamoto, S. Yokoyama, K. Asai and K. Mizuno (2012). "Clinical significance of acid-base balance in an emergency setting in patients with acute heart failure". Journal of Cardiology, 2012, Vol. 60 (3-4), pp: 288-294. doi: 10.1016/j.jjcc.2012.06.004.
[2] K. Sharandeep; K. Praveen; K. Virendra; S. S. Kumar and K. Arun (2013). "Etiology and Prognostic Factors of Acute Liver Failure in Children”. Indian Pediatrics, 07/2013, Vol. 50, Issue 7, pp: 677-679. doi: 10.1007/s13312-013-0189-7.

[3] A. Hany, L. Meno, W. Jonathan, P. Elvira, D. Alan and S. Charles (1997). "Partial Liquid Ventilation (PLV) and Lung Injury: Is PLV Able to Modify Pulmonary Vascular Resistance?" Journal of Pediatric Surgery, 1997, Vol. 32, No. 2, pp: 197-202. doi: 10.1016/S0022-3468(97)90178-0.

[4] F. Intven, M. v. d. Boogaard, P. Pickkers, M. Moviat, P. v. d. Voort and H. v. d. Hoeven (2013). "Stewart analysis of apparently normal acid-base state in the critically ill". Journal of Critical Care, 2013, vol. 28, No. 6, pp: 1048-1054. doi: 10.1016/j.jcrc.2013.06.005.

[5] P. S. Kumar, Y. S. Singh, H. P. Singh and N. Bajaj (2014). "To Study PH Disorders in Severe Acute Malnutrition". Journal of Evolution of Medical and Dental Sciences, 13/10/2014; Vol. 3, Issue 52, pp: 12132-12139. doi: $10.14260 /$ jemds/2014/3602.

[6] H. Handman, K. RaisBahrami, O. Rivera, W. R. Seale and B. L. Short (1997). "Use of intratracheal pulmonary ventilation versus conventional ventilation in meconium aspiration syndrome in a newborn pig model". Critical Care Medicine, 1997, Vol. 25, No. 12, pp: 2025-2030.

[7] B. M. Natalia and W. D. Steven (2015). "Amphetamine-Like Analogues in Diabetes: Speeding towards Ketogenesis". Case Reports in Endocrinology, 2015 (2015), pp: 1-4. doi. $10.1155 / 2015 / 917869$.

[8] C. Barfod, M. M. P. Lauritzen, J. K. Danker, G. Soletormos, P. A. Berlac, F. Lippert, L. H. Lundstrom, K. Antonsen and K. H. W. Lange (2012). "The formation and design of the 'Acute Admission Database'-a database including a prospective, observational cohort of 6279 patients triaged in the emergency department in a larger Danish hospital". Scandinavian Journal of Trauma, Resuscitation and Emergency Medicine, 2012, 20 (29), pp: 1-8.

[9] L. L. Zhu, K. L. Tee, D. Roccatano, B. Sonmez, Y. Ni, Z. H. Sun and U. Schwaneberg (2010). "Directed Evolution of an Antitumor Drug (Arginine Deiminase PpADI) for Increased Activity at Physiological pH". Chembiochem: a European journal of chemical biology, 2010, Vol. 11, No. 5, pp: 691-697. doi: 10.1002/cbic.200900717.

[10] L. L. Zhu, R. Verma, D. Roccatano, Y. Ni, Z. H. Sun and U. Schwaneberg (2010). "A Potential Antitumor Drug (Arginine Deiminase) Reengineered for Efficient Operation under Physiological Conditions". Journal Of Toxicology-Clinical Toxicology, 2010, Vol. 11, No. 16, pp: 2294-2301. doi: 10.1002/cbic.201000458.

[11] K. Robert, K. Rainer, M. Stanislao, S. Torsten and K. M. Detlef (2013). "Regional Citrate Anticoagulation for High Volume Continuous Venovenous Hemodialysis in Surgical Patients With High Bleeding Risk". Therapeutic Apheresis and Dialysis, 2013, Vol. 17, No. 2, pp: 202-212.

[12] B. Mona, I. Arkady, T. Alexander, S. Mordechai, B. Haim; I. Alexander, S. Peter, E. Shmuel, M. Michael and E. Tiberiu (2011). "Perioperative metabolic alkalemia is more frequent than metabolic acidemia in major elective abdominal surgery". Journal of Clinical Monitoring and Computing, 2011, Vol. 25, No. 4, pp: 223-230. 
[13] Z. K. Saritas, M. Korkmaz, T. B. Saritas and R. G. Sivaci, (2014). "Comparison of the Depth of Anesthesia Produced with Dexmedetomidine-Sevoflurane or Medetomidine-Sevoflurane by Using Bispectral Index Monitoring”. Acta Scientiae Veterinariae, 2014, Vol. 42, No. 7, pp: $1-7$.

[14] D. C. Stevens, J. P. Campbell, J. E. Carter and W. A. Watson, (1994). "Acid-Base Abnormalities Associated With Cocaine Toxicity In Emergency Department Patients". Journal Of Toxicology-Clinical Toxicology, 1994, Vol.32, No.1, pp: 31-39.

[15] Y. S. Zhang (1993). "Multilateral Matrix Theory". Beijing:Chinese Statistics Press, 1993.

[16] Y. S. Zhang (2007). "Multilateral System Theory". http://www.mlmatrix.com, 2007.

[17] Y. S. Zhang (2011). "Mathematical reasoning of treatment principle based on Yin Yang Wu Xing theory in traditional Chinese medicine", Chinese Medicine, 2011, Vol. 2, No. 1, pp: 6-15. doi: $10.4236 / \mathrm{cm} .2011 .21002$.

[18] Y. S. Zhang (2011). "Mathematical reasoning of treatment principle based on Yin Yang Wu Xing theory in traditional Chinese medicine (II)", Chinese Medicine, 2011, Vol. 2, No. 4, pp: 158-170. doi: 10.4236/cm.2011.24026.
[19] Y. S. Zhang (2012) "Mathematical reasoning of treatment principle based on the stable logic analysis model of complex systems", Intelligent control and automation, 2012, Vol. 3, No. 1, pp: 6-15. doi: 10.4236/ica.2012.31001.

[20] Y. S. Zhang and W. L. Shao (2012). "Image mathematics-mathematical intervening principle based on Yin Yang Wu Xing theory in traditional Chinese mathematics (I)", Applied Mathematics, 2012, Vol. 3, No. 2, pp: 617-636. doi: 10.4236/am.2012.36096.

[21] Z. Q. Zhang and Y. S. Zhang (2013). "Mathematical reasoning of economic intervening principle based on Yin Yang Wu Xing theory in traditional Chinese economics (I)", Modern Economics, 2013, Vol. 4, pp: 130-144. doi: 10.4236/me.2013.42016.

[22] N. Q. Feng, Y. H. Qiu, F. Wang, Y. S. Zhang and S. Q. Yin (2005). "A logic analysis model about complex system's stability: enlightenment from nature". Lecture Notes in Computer Science, 2005, Vol. 3644, pp: 828-838. doi.org/10.1007/11538059_86.

[23] Y. S. Zhang and B. B. Zhang (2019). "Acupuncture Treating Dystrophy Based on $\mathrm{pH}$. -Mathematical Reasoning of Treatment Principle Based on Yin Yang Wu Xing Theory in Traditional Chinese Medicine (III)". Chinese Medicine, 10, 39-105. doi.org/10.4236/cm.2019.102005. 